ESAIM: M2AN

Vol. 41, No 4, 2007, pp. 771-799

DOI: $10.1051 / \mathrm{m} 2 \mathrm{an}: 2007041$
ESAIM: Mathematical Modelling and Numerical Analysis

www.esaim-m2an.org

\title{
INFLUENCE OF BOTTOM TOPOGRAPHY ON LONG WATER WAVES
}

\author{
Florent Chazel $^{1}$
}

\begin{abstract}
We focus here on the water waves problem for uneven bottoms in the long-wave regime, on an unbounded two or three-dimensional domain. In order to derive asymptotic models for this problem, we consider two different regimes of bottom topography, one for small variations in amplitude, and one for strong variations. Starting from the Zakharov formulation of this problem, we rigorously compute the asymptotic expansion of the involved Dirichlet-Neumann operator. Then, following the global strategy introduced by Bona et al. [Arch. Rational Mech. Anal. 178 (2005) 373-410], we derive new symetric asymptotic models for each regime. The solutions of these systems are proved to give good approximations of solutions of the water waves problem. These results hold for solutions that evanesce at infinity as well as for spatially periodic ones.
\end{abstract}

Mathematics Subject Classification. 76B15, 35L55, 35C20, 35Q35.

Received December 4, 2005. Revised May 7, 2007.

\section{INTRODUCTION}

\section{Generalities}

This paper deals with the water waves problem for uneven bottoms which consists in describing the motion of the free surface and the evolution of the velocity field of a layer of fluid, under the following assumptions: the fluid is ideal, incompressible, irrotationnal, and under the only influence of gravity.

Earlier works have set a good theoretical background for this problem: its well-posedness has been discussed among others by Nalimov [24], Yoshihara [32], Craig [11], Wu [30,31], and Lannes [19].

Nevertheless, the solutions of these equations are very difficult to describe, because of the complexity of these equations. At this point, a classical method is to choose an asymptotic regime, in which we look for approximate models and hence for approximate solutions. We consider in this paper the so-called long-wave regime, where the ratio of the typical amplitude of the waves over the mean depth and the ratio of the square of the mean depth over the square of the typical wave-length are both neglictible compared to 1 and of the same order.

In 2002, Bona et al. constructed in [7] a large class of systems for this regime and performed a formal study in the two-dimensional case. A significant step forward has been made in 2005 by Bona et al. in [8]; they rigorously justified the systems of Bona et al. [8] and derived a new specific class of symmetric systems. The solutions of these systems are proved to converge towards to the solutions of the water waves problem on a large time scale, as the amplitude becomes small and the wavelength large. Thanks to the symmetric structure of

Keywords and phrases. Water waves, uneven bottoms, bottom topography, long-wave approximation, asymptotic expansion, hyperbolic systems, Dirichlet-Neumann operator.

1 Laboratoire de Mathématiques Appliquées de Bordeaux, Université Bordeaux 1, 351 Cours de la Libération, 33405 Talence

Cedex, France. florent.chazel@math.u-bordeaux1.fr

(c) EDP Sciences, SMAI 2007 Article published by EDP Sciences and available at http://www.esaim-m2an.org or http://dx.doi.org/10.1051/m2an:2007041 
these systems, computing solutions is significantly easier than for the water waves problem. Another significant work in this field is due to Lannes and Saut [20], where weakly transverse Boussinesq systems are derived.

However, all these results only hold for flat bottoms. The case of uneven bottoms has been less investigated; some of the significant references are Peregrine [26], Madsen et al. [21], Nwogu [25], and Chen [10]. Peregrine was the first one to formulate the classical Boussinesq equations for waves in shallow water with variable depth on a three-dimensionnal domain. Following this work, Madsen et al. and Nwogu derived new Boussinesq-like systems for uneven bottoms with improved linear dispersion properties. Recently, Chen performed a formal study of the water waves problem for uneven bottoms with small variations in amplitude, in 1D of surface, and derived a class of asymptotic models inspired by the work of Bona, Chen and Saut. To our knowledge, the only rigorously justified result on the uneven bottoms case is the work of Iguchi [15], who provided a rigorous approximation via a system of KdV-like equations, in the case of a slowly varying bottom.

The main idea of our paper is to reconsider the water waves problem for uneven bottoms in the angle shown by Bona et al. [8]. Moreover, our goal is to consider two different types of bottoms: bottoms with small variations in amplitude, and bottoms with strong variations in amplitude. To this end, we introduce a new parameter to characterize the shape of the bottom. In the end, new asymptotic models are derived, studied and rigorously justified under the assumption that large time solutions to the water waves equations exist and remain bounded ${ }^{1}$.

\section{Presentation and formulation of the problem}

In this paper, we work indifferently in two or three dimensions. Let us denote by $X \in \mathbb{R}^{d}$ the transverse variable, $d$ being equal to 1 or 2 . In the two-dimensional case, $d=1$ and $X=x$ corresponds to the coordinate along the primary direction of propagation whilst in the three-dimensional case, $d=2$ and $X=(x, y)$ represents the horizontal variables. We restrict our study to the case where the free surface and the bottom can be described by the graph of two functions $(t, X) \rightarrow \eta(t, X)$ and $X \rightarrow b(X)$ defined respectively over the surface $z=0$ and the mean depth $z=-h_{0}$ both at the steady state, $t$ corresponding to the time variable. The time-dependant domain $\Omega_{t}$ of the fluid is thus taken of the form:

$$
\Omega_{t}=\left\{(X, z), X \in \mathbb{R}^{d},-h_{0}+b(X) \leq z \leq \eta(t, X)\right\} .
$$

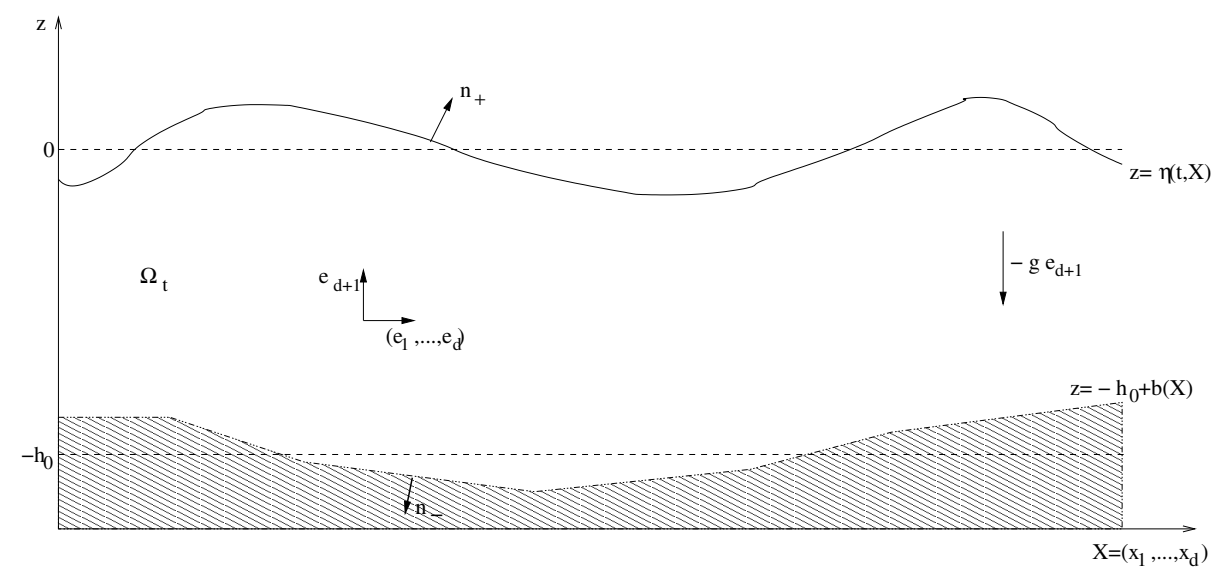

FIGURE 1. Sketch of the domain.

\footnotetext{
${ }^{1}$ Such an assumption has been recently proved in [2] and the mathematical justification of the Boussinesq-like models derived here is therefore complete. Note that other well-known models - like Green-Naghdi, Serre, full-dispersion, etc. — are also fully justified in this work.
} 
In order to avoid some special physical cases such as the presence of islands or beaches, we set a condition of minimal water depth: there exists a strictly positive constant $h_{\min }$ such that

$$
\eta(t, X)+h_{0}-b(X) \geq h_{\min }, \quad(t, X) \in \mathbb{R} \times \mathbb{R}^{2}
$$

For the sake of simplicity, we assume here that $b$ and all its derivatives are bounded.

The motion of the fluid is described by the following system of equations:

$$
\left\{\begin{array}{cl}
\partial_{t} V+V \cdot \nabla_{X, z} V=-g e_{z}-\nabla_{X, z} P & \text { in } \Omega_{t}, t \geq 0, \\
\nabla_{X, z} \cdot V=0 & \text { in } \Omega_{t}, \quad t \geq 0, \\
\nabla_{X, z} \times V=0 & \text { in } \Omega_{t}, t \geq 0, \\
\partial_{t} \eta-\left.\sqrt{1+\left|\nabla_{X} \eta\right|^{2}} \mathbf{n}_{+} \cdot V\right|_{z=\eta(t, X)}=0 & \text { for } t \geq 0, X \in \mathbb{R}^{d}, \\
P_{\left.\right|_{z=\eta(t, X)}}=0 & \text { for } t \geq 0, X \in \mathbb{R}^{d}, \\
\left.\mathbf{n}_{-} \cdot V\right|_{z=-h_{0}+b(X)}=0 & \text { for } t \geq 0, X \in \mathbb{R}^{d},
\end{array}\right.
$$

where $\mathbf{n}_{+}=\frac{1}{\sqrt{1+|\nabla \eta|^{2}}}(-\nabla \eta, 1)^{T}$ denotes the outward normal vector to the surface and $\mathbf{n}_{-}=\frac{1}{\sqrt{1+|\nabla b|^{2}}}(\nabla b,-1)^{T}$ denotes the outward normal vector to the bottom. The first equation corresponds to the Euler equation for a perfect fluid under the influence of gravity (which is characterized by the term $-g e_{z}$ where $e_{z}$ denotes the unit vector along the vertical component). The second and third one express the incompressibility and irrotationnality of the fluid. The fourth and last ones are the boundary conditions at the surface and the bottom; they state that no fluid particle crosses these surfaces. Finally, we neglect the surface tension effects and assume that the pressure $\mathrm{P}$ is constant at the surface: up to a renormalization, we can assume that this constant is equal to 0 .

In this paper, we use the Bernoulli formulation of the water-waves equations. The conditions of incompressibility and irrotationnality ensure the existence of a potential flow $\phi$ such that $V=\nabla_{X, z} \phi$ and $\Delta_{X, z} \phi=0$. From now one we use the following notations: $\nabla=\nabla_{X}$ and $\Delta=\Delta_{X}$. Written in terms of the velocity potential, equations (0.2) read

$$
\left\{\begin{array}{cl}
\partial_{t} \phi+\frac{1}{2}\left[|\nabla \phi|^{2}+\left|\partial_{z} \phi\right|^{2}\right]+g z=-P & \text { in } \Omega_{t}, \quad t \geq 0, \\
\Delta \phi+\partial_{z}^{2} \phi=0 & \text { in } \Omega_{t}, \quad t \geq 0, \\
\partial_{t} \eta-\left.\sqrt{1+|\nabla \eta|^{2}} \partial_{\mathbf{n}_{+}} \phi\right|_{z=\eta(t, X)}=0 & \text { for } t \geq 0, X \in \mathbb{R}^{d}, \\
\left.\partial_{\mathbf{n}_{-}} \phi\right|_{z=-h_{0}+b(X)}=0 & \text { for } t \geq 0, X \in \mathbb{R}^{d},
\end{array}\right.
$$

where we used the notations $\partial_{\mathbf{n}_{-}}=\mathbf{n}_{-} \cdot\left(\begin{array}{c}\nabla \\ \partial_{z}\end{array}\right)$ and $\partial_{\mathbf{n}_{+}}=\mathbf{n}_{+} \cdot\left(\begin{array}{c}\nabla \\ \partial_{z}\end{array}\right)$. 
Making the dependence on the $\mathrm{X}$ and $z$ explicit in the boundary conditions and taking the trace of $(0.3)$ on the free surface yields

$$
\left\{\begin{array}{cl}
\Delta \phi+\partial_{z}^{2} \phi=0 & \text { in } \Omega_{t}, t \geq 0, \\
\partial_{t} \phi+\frac{1}{2}\left[|\nabla \phi|^{2}+\left|\partial_{z} \phi\right|^{2}\right]+g \eta=0 & \text { at } z=\eta(t, X), X \in \mathbb{R}^{d}, t \geq 0, \\
\partial_{t} \eta+\nabla \eta \cdot \nabla \phi-\partial_{z} \phi=0 & \text { at } z=\eta(t, X), X \in \mathbb{R}^{d}, t \geq 0, \\
\nabla b \cdot \nabla \phi-\partial_{z} \phi=0 & \text { at } z=-h_{0}+b(X), X \in \mathbb{R}^{d}, t \geq 0 .
\end{array}\right.
$$

We dimensionalize these equations using the following quantities: $\lambda$ is the typical wavelength, $a$ the typical amplitude of the waves, $h_{0}$ the mean depth of the fluid, $b_{0}$ the typical amplitude of the bottom, $t_{0}=\frac{\lambda}{\sqrt{g h_{0}}} \mathrm{a}$ typical period of time $\left(\sqrt{g h_{0}}\right.$ corresponding to sound velocity in the fluid $)$ and $\phi_{0}=\frac{\lambda a}{h_{0}} \sqrt{g h_{0}}$. Introducing the following parameters:

$$
\epsilon=\frac{a}{h_{0}} ; \quad \beta=\frac{b_{0}}{h_{0}} ; \quad S=\frac{a \lambda^{2}}{h_{0}^{3}}
$$

and taking the Stokes number $S$ to be equal to one, one gets the following non-dimensionalized version of (0.4):

$$
\left\{\begin{array}{cl}
\varepsilon \Delta \phi+\partial_{z}^{2} \phi=0 & -1+\beta b \leq z \leq \varepsilon \eta, X \in \mathbb{R}^{d}, t \geq 0, \\
\partial_{t} \phi+\frac{1}{2}\left[\varepsilon|\nabla \phi|^{2}+\left|\partial_{z} \phi\right|^{2}\right]+g \eta=0 & \text { at } z=\varepsilon \eta, X \in \mathbb{R}^{d}, t \geq 0, \\
\partial_{t} \eta+\varepsilon \nabla \eta \cdot \nabla \phi-\frac{1}{\varepsilon} \partial_{z} \phi=0 & \text { at } z=\varepsilon \eta, X \in \mathbb{R}^{d}, t \geq 0, \\
\partial_{z} \phi-\varepsilon \beta \nabla b \cdot \nabla \phi=0 & \text { at } z=-1+\beta b, X \in \mathbb{R}^{d}, t \geq 0 .
\end{array}\right.
$$

The final step consists in giving the Zakharov formulation corresponding to (0.5). To this end, we introduce the trace of the velocity potential $\phi$ at the free surface, namely $\psi$ :

$$
\psi(t, X)=\phi(t, X, \varepsilon \eta(t, X))
$$

and the operator $Z_{\varepsilon}(\varepsilon \eta, \beta b)$ which maps $\psi$ to $\left.\partial_{z} \phi\right|_{z=\varepsilon \eta}$. This operator is defined for any $f \in W^{1, \infty}\left(\mathbb{R}^{d}\right)$ by:

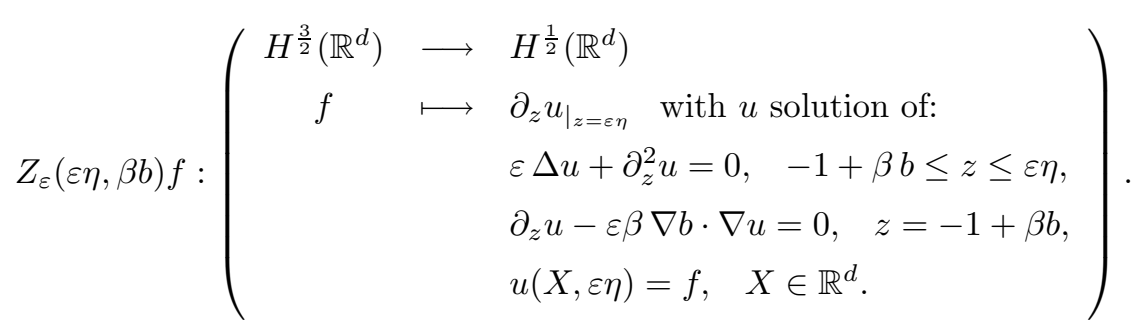


Using this operator and computing the derivatives of $\psi$ in terms of $\psi$ and $\eta$, the final formulation $\left(S_{0}\right)$ of the water waves problem reads:

$$
\left(S_{0}\right)\left\{\begin{array}{l}
\partial_{t} \psi-\varepsilon \partial_{t} \eta Z_{\varepsilon}(\varepsilon \eta, \beta b) \psi+\frac{1}{2}\left[\varepsilon\left|\nabla \psi-\varepsilon \nabla \eta Z_{\varepsilon}(\varepsilon \eta, \beta b) \psi\right|^{2}+\left|Z_{\varepsilon}(\varepsilon \eta, \beta b) \psi\right|^{2}\right]+\eta=0 \\
\partial_{t} \eta+\varepsilon \nabla \eta \cdot\left[\nabla \psi-\varepsilon \nabla \eta Z_{\varepsilon}(\varepsilon \eta, \beta b) \psi\right]=\frac{1}{\varepsilon} Z_{\varepsilon}(\varepsilon \eta, \beta b) \psi
\end{array}\right.
$$

\section{Organization of the paper}

The aim of this paper is to derive and study two different asymptotic regimes based each on a specific assumption on the parameter $\beta$ which characterizes the topography of the bottom. The first regime corresponds to the case $\beta=O(\varepsilon)$ where the amplitude of the bottom variations is small. The second one deals with the more complex case $\beta=O(1)$ which corresponds to bottoms with large variations in amplitude.

The following section is devoted to the asymptotic expansion of the operator $Z_{\varepsilon}(\varepsilon \eta, \beta b)$ in the two regimes mentionned above. To this end, we use a general method to rigorously derive asymptotic expansions of DirichletNeumann operators for a large class of elliptic problems. This result is then applied in each regime, wherein a formal expansion is performed and an asymptotic Boussinesq-like model of (0.7) is derived. The second and third sections of this paper are both devoted to the derivation of new classes of equivalent systems, following the strategy developped in [8]. In the end, completely symmetric systems are obtained for both regimes: convergence results are proved, showing that solutions of these symmetric asymptotic systems converge to exact solutions of the water waves problem.

\section{Asymptotic expansion of the operator $Z_{\varepsilon}(\varepsilon \eta, \beta b)$}

This section is devoted to the asymptotic expansion as $\varepsilon$ goes to 0 of the operator $Z_{\varepsilon}(\varepsilon \eta, \beta b)$ defined in the previous section, in both regimes $\beta=O(\varepsilon)$ and $\beta=O(1)$. To this end, we first state some general results on elliptic equations on a strip: the main theorem provides a general method for determining approximations of Dirichlet-Neumann operators. This result is then applied to the case of the operator $Z_{\varepsilon}(\varepsilon \eta, \beta b)$ and two asymptotic models with bottom effects are derived.

\subsection{Elliptic equations on a strip}

In this part, we study a general elliptic equation on a domain $\Omega$ given by:

$$
\Omega=\left\{(X, z) \in \mathbb{R}^{d+1} / X \in \mathbb{R}^{d},-h_{0}+B(X)<z<\eta(X)\right\}
$$

where the functions $B$ and $\eta$ satisfy the following condition:

$$
\exists h_{\text {min }}>0, \forall X \in \mathbb{R}^{d}, \eta(X)-B(X)+h_{0} \geq h_{m i n} .
$$

Let us consider the following general elliptic boundary value problem set on the domain $\Omega$ :

$$
\begin{gathered}
-\nabla_{X, z} \cdot P \nabla_{X, z} u=0 \quad \text { in } \Omega, \\
u_{\left.\right|_{z=\eta(X)}}=f \text { and }\left.\partial_{n} u\right|_{\left.\right|_{z=-h_{0}+B(X)}}=0,
\end{gathered}
$$

where $P$ is a diagonal $(d+1) \times(d+1)$ matrix whose coefficients $\left(p_{i}\right)_{1 \leq i \leq d+1}$ are constant and strictly positive. Straightforwardly, $P$ is coercive. We denote by $\partial_{n} u_{\mid z=-h_{0}+B(X)}$ the outward conormal derivative associated to $P$ of $u$ at the lower boundary $\left\{z=-h_{0}+B(X)\right\}$, namely:

$$
\partial_{n} u_{\left.\right|_{z=-h_{0}+B(X)}}=-\mathbf{n}_{-} \cdot P \nabla_{X, z} u_{\left.\right|_{z=-h_{0}+B(X)}}
$$


where $\mathbf{n}_{-}$denotes the outward normal vector to the lower boundary of $\Omega$. For the sake of simplicity, the notation $\partial_{n}$ always denotes the outward conormal derivative associated to the elliptic problem under consideration.

Remark 1.1. When no confusion can be made, we denote $\nabla_{X}$ by $\nabla$.

As in $[8,19,23]$ we transform the boundary value problem (1.2)-(1.3) into a new boundary problem defined over the flat band

$$
\mathcal{S}=\left\{(X, z) \in \mathbb{R}^{d+1} / X \in \mathbb{R}^{d},-1<z<0\right\} .
$$

Let $S$ be the following diffeomorphism mapping $\mathcal{S}$ to $\Omega$ :

$$
S:\left(\begin{array}{ccl}
\mathcal{S} & \longrightarrow & \Omega \\
(X, z) & \longmapsto & \left(X, s(X, z)=\left(\eta(X)-B(X)+h_{0}\right) z+\eta(X)\right)
\end{array}\right) .
$$

Remark 1.2. As shown in [19], a more complex "regularizing" diffeomorphism must be used instead of $(\mathcal{S})$ to obtain a shard dependence on $\eta$ in terms of regularity, but since the trivial diffeomorphism $(\mathcal{S})$ suffices for our present purpose, we use it for the sake of simplicity.

Clearly, if $v$ is defined over $\Omega$ then $\underline{v}=v \circ S$ is defined over $\mathcal{S}$. As a consequence, we can set an equivalent problem to (1.2)-(1.3) on the flat band $\mathcal{S}$ using the following proposition (see [18,19] for a proof):

Proposition 1.3. $u$ is solution of (1.2)-(1.3) if and only if $\underline{u}=u \circ S$ is solution of the boundary value problem

$$
\begin{gathered}
-\nabla_{X, z} \cdot \underline{P} \nabla_{X, z} \underline{u}=0 \quad \text { in } \mathcal{S}, \\
\underline{u}_{\mid z=0}=f \quad \text { and } \quad \partial_{n} \underline{u}_{\left.\right|_{z=-1}}=0,
\end{gathered}
$$

where $\underline{P}(X, z)$ is given by

$$
\begin{gathered}
\underline{P}(X, z)=\frac{1}{\eta+h_{0}-B} M^{T} P M \\
\text { with } M(X, z)=\left(\begin{array}{cc}
\left(\eta+h_{0}-B\right) I_{d \times d} & -(z+1) \nabla \eta+z \nabla B \\
0 & 1
\end{array}\right) .
\end{gathered}
$$

Consequently, let us consider boundary value problems belonging to the class (1.5)-(1.6). From now on, all references to the problem set on $\mathcal{S}$ will be labelled with an underscore.

It is well kwnown that elliptic boundary value problems of the form (1.5)-(1.6) are well posed under appropriate assumptions: assuming that $\underline{P}$ and all its derivatives are bounded on $\mathcal{S}$, if $f \in H^{k+\frac{3}{2}}\left(\mathbb{R}^{d}\right)$ then there exists a unique solution $u \in H^{k+2}(\mathcal{S})$ to $(1.5)-(1.6)$. The proof is very classical and we omit it here.

As previously seen, we need to consider the following operator $Z(\eta, B)$ which maps the value of $u$ at the upper bound to the value of $\left.\partial_{z} u\right|_{z=\eta}$ :

$$
Z(\eta, B):\left(\begin{array}{ccc}
H^{\frac{3}{2}}\left(\mathbb{R}^{d}\right) & \longrightarrow & H^{\frac{1}{2}}\left(\mathbb{R}^{d}\right) \\
f & \longmapsto & \left.\partial_{z} u\right|_{z=\eta} \quad \text { with } u \text { solution of }(1.2)-(1.3)
\end{array}\right)
$$

Remark 1.4. The operator $Z_{\varepsilon}$ defined in (0.6) corresponds to the operator $Z$ in the case where $P=\left(\begin{array}{cc}\varepsilon I_{d} & 0 \\ 0 & 1\end{array}\right)$ in $(1.2)-(1.3)$.

To construct an approximation of this operator $Z(\eta, B)$, we need the following lemma which gives a coercitivity result taking into account the anisotropy of (1.2)-(1.3). 
Lemma 1.5. Let $\eta \in W^{1, \infty}\left(\mathbb{R}^{d}\right)$ and $B \in W^{1, \infty}\left(\mathbb{R}^{d}\right)$. Then for all $V \in \mathbb{R}^{d+1}$ :

$$
(V, \underline{P} V) \geq c_{0}\left(\|\eta\|_{W^{1, \infty}},\|B\|_{W^{1, \infty}}\right)|\sqrt{P} V|^{2},
$$

where $c_{0}$ is a strictly positive function given by

$$
c_{0}(x, y)=\frac{h_{\min }}{(d+1)^{2}} \min \left(1, \frac{1}{h_{\min }\left(x+h_{0}+y\right)}, \frac{\min _{1 \leq i \leq d} \frac{p_{d+1}}{p_{i}}}{(x+y)^{2}}\right)
$$

Proof. Using Proposition 1.3, we can write, with $\delta(X)=\eta(X)+h_{0}-B(X)$ :

$$
\begin{aligned}
(V, \underline{P} V) & =\left(\frac{1}{\delta} V, M^{T} P M V\right) \\
& =\left(\frac{1}{\delta} M V, P M V\right) \\
& =\left(\frac{1}{\delta} \sqrt{P} M V, \sqrt{P} M V\right) \\
& =\left|\frac{1}{\sqrt{\delta}} \mathcal{M}(\sqrt{P} V)\right|^{2}
\end{aligned}
$$

where $\mathcal{M}=\sqrt{P} M(\sqrt{P})^{-1}$. Thanks to condition (1.1), we deduce the invertibility of $M$ and hence of $\mathcal{M}$. This yields the following inequality: for all $U \in \mathbb{R}^{d+1}$,

$$
|U| \leq(d+1)\left|\sqrt{\delta} \mathcal{M}^{-1}\right|_{\infty}\left|\frac{1}{\sqrt{\delta}} \mathcal{M} U\right|
$$

with

$$
\mathcal{M}^{-1}=\left(\begin{array}{cc}
\frac{1}{\delta} I_{d \times d} & \frac{1}{\delta \sqrt{p_{d+1}}} \sqrt{P^{d}}((z+1) \nabla \eta-z \nabla B) \\
0 & 1
\end{array}\right),
$$

where $|A|_{\infty}=\sup _{1 \leq i, j \leq d+1}\left|a_{i, j}\right|_{L^{\infty}\left(\mathbb{R}^{d}\right)}$ and $P^{d}$ is the $d \times d$ diagonal matrix whose coefficients are $\left(p_{i}\right)_{1 \leq i \leq d}$.

If we apply the previous inequality to our problem, one gets:

$$
(V, \underline{P} V) \geq \frac{1}{(d+1)^{2}\left|\sqrt{\delta} \mathcal{M}^{-1}\right|_{\infty}^{2}}|\sqrt{P} V|^{2} .
$$

Thanks to the expression of $\mathcal{M}^{-1}$ given above, we obtain the following inequality:

$$
(V, \underline{P} V) \geq c_{0}\left(\|\eta\|_{W^{1, \infty}},\|B\|_{W^{1, \infty}}\right)|\sqrt{P} V|^{2},
$$

where $c_{0}$ as in the statement of the Lemma 1.5 .

Let us introduce the space $H^{k, 0}(\mathcal{S})$ :

$$
H^{k, 0}(\mathcal{S})=\left\{v \in L^{2}(\mathcal{S}),\|v\|_{H^{k, 0}}:=\left(\int_{-1}^{0}|v(\cdot, z)|_{H^{k}\left(\mathbb{R}^{d}\right)}^{2} \mathrm{~d} z\right)^{\frac{1}{2}}<+\infty\right\}
$$


We can now state the main result of this section, which gives a rigourous method for deriving asymptotic expansions of $Z(\eta, B)$. Of course, $P$, and thus $\underline{P}$, as well as the boundaries $\eta$ and $B$, can depend on $\varepsilon$ in the following theorem. In such cases, the proof can be easily adapted just by remembering that $0<\varepsilon<1$.

Theorem 1.6. Let $p \in \mathbb{N}^{*}, k \in \mathbb{N}^{*}, \eta \in W^{k+2, \infty}\left(\mathbb{R}^{d}\right)$ and $B \in W^{k+2, \infty}\left(\mathbb{R}^{d}\right)$. Let $0<\varepsilon<1$ and $u_{\text {app }}$ be such that

$$
\begin{aligned}
& -\nabla_{X, z} \cdot \underline{P} \nabla_{X, z} u_{a p p}=\varepsilon^{p} R^{\varepsilon} \quad \text { in } \mathcal{S}, \\
& u_{\left.a p p\right|_{z=0}}=f,\left.\quad \partial_{n} u_{a p p \mid}\right|_{z=-1}=\varepsilon^{p} r^{\varepsilon},
\end{aligned}
$$

where $\left(R^{\varepsilon}\right)_{0<\varepsilon<1}$ and $\left(r^{\varepsilon}\right)_{0<\varepsilon<1}$ are bounded independently of $\varepsilon$ respectively in $H^{k+1,0}(\mathcal{S})$ and $H^{k+1}\left(\mathbb{R}^{d}\right)$.

Assuming that $h_{\text {min }}$ is independent of $\varepsilon$ and that the coefficients $\left(p_{i}\right)_{1 \leq i \leq d+1}$ of $P$ are such that $\left(\frac{p_{i}}{p_{d+1}}\right)_{1 \leq i \leq d}$ are bounded by a constant $\gamma$ independent of $\varepsilon$, we have

$$
\left|Z(\eta, B) f-\frac{1}{\eta+h_{0}-B}\left(\partial_{z} u_{a p p}\right)_{\left.\right|_{z=0}}\right|_{H^{k+\frac{1}{2}}} \leq \frac{\varepsilon^{p}}{\sqrt{p_{d+1}}} C_{k+2}\left(|| R^{\varepsilon} \|_{H^{k+1,0}}+\left|r^{\varepsilon}\right|_{H^{k+1}}\right),
$$

where $C_{k+2}=C\left(|\eta|_{W^{k+2, \infty}},|B|_{W^{k+2, \infty}}\right)$ and $C$ is a non decreasing function of its arguments, independent of the coefficients $\left(p_{i}\right)_{1 \leq i \leq d+1}$.

Proof. In this proof, we often use the notation $C_{k}=C\left(|\eta|_{W^{k, \infty}},|B|_{W^{k, \infty}}, h_{0}, h_{m i n}, k, d, \gamma\right)$ where $\mathrm{C}$ is a nondecreasing function of its arguments. The notation $C_{k}$ can thus refer to different constants, but of the same kind.

A simple computation shows that $Z(\eta, B)$ can be expressed in terms of the solution $u$ of (1.5)-(1.6) via the following relation:

Using this fact, we can write

$$
Z(\eta, B) f=\frac{1}{\eta+h_{0}-B} \partial_{z} \underline{u}_{z=0} .
$$

$$
Z(\eta, B) f-\left.\frac{1}{\eta+h_{0}-B} \partial_{z} u_{a p p \mid}\right|_{z=0}=\frac{1}{\eta+h_{0}-B} \partial_{z}\left(\underline{u}-u_{a p p}\right)_{\left.\right|_{z=0}} .
$$

Introducing $\varphi:=u_{a p p}-\underline{u}$ we use a trace theorem (see Métivier [22], pp. 23-27) to get

$$
\left|Z(\eta, B) f-\frac{1}{\eta+h_{0}-B}\left(\partial_{z} u_{a p p}\right)_{\mid z=0}\right|_{H^{k+\frac{1}{2}}} \leq C_{k+1}\left(\left\|\partial_{z} \varphi\right\|_{H^{k+1,0}}+\left\|\partial_{z}^{2} \varphi\right\|_{H^{k, 0}}\right) .
$$

It is clear that the proof relies on finding an adequate control of $\left\|\partial_{z} \varphi\right\|_{H^{k+1,0}}$ and $\left\|\partial_{z}^{2} \varphi\right\|_{H^{k, 0}}$. The rest of this proof will hence be devoted to the estimate of both terms.

1. Let us begin with the estimate of $\left\|\partial_{z} \varphi\right\|_{H^{k+1,0}}$. To deal correctly with this problem, we introduce the following norm $\|\cdot\|_{\dot{H}^{1}}$ defined by:

$$
\|\varphi\|_{\dot{H}^{1}}:=\left\|\sqrt{P} \nabla_{X, z} \varphi\right\|_{L^{2}(\mathcal{S})} .
$$

First remark that for all $\alpha \in \mathbb{N}^{d}$ such that $|\alpha| \leq k, \partial^{\alpha} \varphi$ solves:

$$
\left\{\begin{array}{l}
-\nabla_{X, z} \cdot \underline{P} \nabla_{X, z} \partial^{\alpha} \varphi=\varepsilon^{p} \partial^{\alpha} R^{\varepsilon}+\nabla_{X, z} \cdot\left[\partial^{\alpha}, \underline{P}\right] \nabla_{X, z} \varphi \\
\partial^{\alpha} \varphi_{\mid z=0}=0, \quad \partial_{n}\left(\partial^{\alpha} \varphi\right)_{\mid z=-1}+\partial_{n}^{\left[\partial^{\alpha}, \underline{P}\right]} \varphi_{z=-1}=\varepsilon^{p} \partial^{\alpha} r^{\varepsilon} .
\end{array}\right.
$$

In order to get an adequate control of the norm $\left\|\partial_{z} \varphi\right\|_{H^{k+1,0}}$, we prove the following estimate by induction on $|\alpha| \leq k$ :

$$
\forall \alpha \in \mathbb{N}^{d} /|\alpha| \leq k, \quad\left\|\partial^{\alpha} \varphi\right\|_{\dot{H}^{1}} \leq \frac{\varepsilon^{p}}{\sqrt{p_{d+1}}} C_{k+1}\left(\left\|R^{\varepsilon}\right\|_{H^{k, 0}}+\left|r^{\varepsilon}\right|_{H^{k}}\right) .
$$

The proof of (1.11) is hence divided into two parts: initialization of the induction and heredity. 
- Initialization: $|\alpha|=0$.

Taking $\alpha=0$, multiplying (1.10) by $\varphi$ and integrating by parts leads to:

$$
\left(\underline{P} \nabla_{X, z} \varphi, \nabla_{X, z} \varphi\right)_{L^{2}(\mathcal{S})}+\int_{\mathbb{R}^{d}} \partial_{n} \varphi_{\left.\right|_{z=0}} \varphi_{\left.\right|_{z=0}}-\int_{\mathbb{R}^{d}} \partial_{n} \varphi_{\left.\right|_{z=-1}} \varphi_{\left.\right|_{z=-1}}=\left(\varepsilon^{p} R^{\varepsilon}, \varphi\right)_{L^{2}(\mathcal{S})} .
$$

The boundary term at the free surface vanishes because of the condition $\varphi_{\mid z=0}=0$ and using the condition at the bottom leads to:

$$
\left(\underline{P} \nabla_{X, z} \varphi, \nabla_{X, z} \varphi\right)_{L^{2}(\mathcal{S})}=\left(\varepsilon^{p} R^{\varepsilon}, \varphi\right)_{L^{2}(\mathcal{S})}+\varepsilon^{p} \int_{\mathbb{R}^{d}} r^{\varepsilon} \varphi_{z=-1}
$$

Finally, using Cauchy-Schwarz inequality, one gets:

$$
\left(\underline{P} \nabla_{X, z} \varphi, \nabla_{X, z} \varphi\right)_{L^{2}(\mathcal{S})} \leq \varepsilon^{p}\left\|R^{\varepsilon}\right\|_{L^{2}(\mathcal{S})}\|\varphi\|_{L^{2}(\mathcal{S})}+\varepsilon^{p}\left|r^{\varepsilon}\right|_{L^{2}\left(\mathbb{R}^{d}\right)}\left|\varphi_{\left.\right|_{z=-1}}\right|_{L^{2}\left(\mathbb{R}^{d}\right)} .
$$

Recalling that $\varphi_{\left.\right|_{z=0}}=0$ and that the band $\mathcal{S}$ is bounded in the vertical direction, one can use Poincaré inequality so that $\|\varphi\|_{L^{2}(\mathcal{S})} \leq\left\|\partial_{z} \varphi\right\|_{L^{2}(\mathcal{S})}$ and $\left.|\varphi|_{z=-1}\right|_{L^{2}\left(\mathbb{R}^{d}\right)} \leq\left\|\partial_{z} \varphi\right\|_{L^{2}(\mathcal{S})}$. Therefore, (1.12) yields

$$
\left(\underline{P} \nabla_{X, z} \varphi, \nabla_{X, z} \varphi\right)_{L^{2}(\mathcal{S})} \leq \frac{\varepsilon^{p}}{\sqrt{p_{d+1}}}|| R^{\varepsilon}\left\|_{L^{2}(\mathcal{S})}\right\| \varphi\left\|_{\dot{H}^{1}}+\frac{\varepsilon^{p}}{\sqrt{p_{d+1}}}\left|r^{\varepsilon}\right|_{L^{2}\left(\mathbb{R}^{d}\right)}\right\| \varphi \|_{\dot{H}^{1}} .
$$

Using Lemma 1.5 to bound $\left(\underline{P} \nabla_{X, z} \varphi, \nabla_{X, z} \varphi\right)_{L^{2}(\mathcal{S})}$ from below, one finally gets:

$$
c_{0}\left(|\eta|_{W^{1, \infty}},|B|_{W^{1, \infty}}\right)\|\varphi\|_{\dot{H}^{1}}^{2} \leq \frac{\varepsilon^{p}}{\sqrt{p_{d+1}}}\left(\left\|R^{\varepsilon}\right\|_{H^{0,0}}+\left|r^{\varepsilon}\right|_{H^{0}}\right)\|\varphi\|_{\dot{H}^{1}} .
$$

Since $c_{0}\left(|\eta|_{W^{1, \infty}},|B|_{W^{1, \infty}}\right)$ depends only on $h_{m i n}, d$ and $\gamma$ through the quantity $\min _{1 \leq i \leq d} \frac{p_{d+1}}{p_{i}}$ (by Lem. 1.5), and since the function $c_{0}$ is a decreasing function of its arguments (again by Lem. 1.5), we get the following estimate:

which ends the initialization of the induction.

$$
\|\varphi\|_{\dot{H}^{1}} \leq \frac{\varepsilon^{p}}{\sqrt{p_{d+1}}} C_{1}\left(\left\|R^{\varepsilon}\right\|_{H^{0,0}}+\left|r^{\varepsilon}\right|_{H^{0}}\right)
$$

- Heredity: for $m \in \mathbb{N}^{*}$ fixed such that $m \leq k$, we suppose that (1.11) is verified for all $\alpha \in \mathbb{N}^{d}$ such that $|\alpha| \leq m-1$.

Let $\alpha \in \mathbb{N}^{d}$ such that $|\alpha|=m$. Multiplying (1.10) by $\partial^{\alpha} \varphi$ and integrating by parts on $\mathcal{S}$ leads to:

$$
\begin{gathered}
\left(\underline{P} \nabla_{X, z} \partial^{\alpha} \varphi, \nabla_{X, z} \partial^{\alpha} \varphi\right)_{L^{2}(\mathcal{S})}+\int_{\mathbb{R}^{d}} \partial^{\alpha} \varphi_{\mid z=0} \partial_{n} \partial^{\alpha} \varphi_{\mid z=0}-\int_{\mathbb{R}^{d}} \partial^{\alpha} \varphi_{\mid z=-1} \partial_{n} \partial^{\alpha} \varphi_{\mid z=-1}=\left(\varepsilon^{p} \partial^{\alpha} R^{\varepsilon}, \partial^{\alpha} \varphi\right)_{L^{2}(\mathcal{S})} \\
-\left(\left[\partial^{\alpha}, \underline{P}\right] \nabla_{X, z} \varphi, \nabla_{X, z} \partial^{\alpha} \varphi\right)_{L^{2}(\mathcal{S})}-\int_{\mathbb{R}^{d}} \partial^{\alpha} \varphi_{\mid z=0} \partial_{n}^{\left[\partial^{\alpha}, \underline{P}\right]} \varphi_{\mid z=0}+\int_{\mathbb{R}^{d}} \partial^{\alpha} \varphi_{\mid z=-1} \partial_{n}^{\left[\partial^{\alpha}, \underline{P}\right]} \varphi_{\mid z=-1}
\end{gathered}
$$

The boundary terms at $z=0$ vanish because of the condition $\partial^{\alpha} \varphi_{\mid z=0}=0$, and using the second boundary condition $\partial_{n}\left(\partial^{\alpha} \varphi\right)_{\mid z=-1}+\partial_{n}^{\left[\partial^{\alpha}, \underline{P}\right]} \varphi_{\mid z=-1}=\varepsilon^{p} \partial^{\alpha} r^{\varepsilon}$, one gets:

$$
\left(\underline{P} \nabla_{X, z} \partial^{\alpha} \varphi, \nabla_{X, z} \partial^{\alpha} \varphi\right)_{L^{2}}=\left(\varepsilon^{p} \partial^{\alpha} R^{\varepsilon}, \partial^{\alpha} \varphi\right)_{L^{2}}+\varepsilon^{p} \int_{\mathbb{R}^{d}} \partial^{\alpha} \varphi_{\mid z=-1} \partial^{\alpha} r^{\varepsilon}-\left(\left[\partial^{\alpha}, \underline{P}\right] \nabla_{X, z} \varphi, \nabla_{X, z} \partial^{\alpha} \varphi\right)_{L^{2}}
$$

and with Cauchy-Schwarz:

$$
\begin{aligned}
\left(\underline{P} \nabla_{X, z} \partial^{\alpha} \varphi, \nabla_{X, z} \partial^{\alpha} \varphi\right)_{L^{2}(\mathcal{S})} \leq & \varepsilon^{p}|| \partial^{\alpha} R^{\varepsilon}\left\|_{L^{2}(\mathcal{S})}|| \partial^{\alpha} \varphi\right\|_{L^{2}(\mathcal{S})}+\varepsilon^{p}\left|\partial^{\alpha} r^{\varepsilon}\right|_{L^{2}\left(\mathbb{R}^{d}\right)}\left|\partial^{\alpha} \varphi_{\left.\right|_{z=-1}}\right|_{L^{2}\left(\mathbb{R}^{d}\right)} \\
& +\left|\left(\left[\partial^{\alpha}, \underline{P}\right] \nabla_{X, z} \varphi, \nabla_{X, z} \partial^{\alpha} \varphi\right)_{L^{2}(\mathcal{S})}\right| .
\end{aligned}
$$


By using the same method and arguments as in the initialization, the following inequality arises:

$$
c_{0}\left(|\eta|_{W^{1, \infty}},|B|_{W^{1, \infty}}\right)\left\|\partial^{\alpha} \varphi\right\|_{\dot{H}^{1}}^{2} \leq \frac{\varepsilon^{p}}{\sqrt{p_{d+1}}}\left(\left\|R^{\varepsilon}\right\|_{H^{k, 0}}+\left|r^{\varepsilon}\right|_{H^{k}}\right)\left\|\partial^{\alpha} \varphi\right\|_{\dot{H}^{1}}+\left|\left(\left[\partial^{\alpha}, \underline{P}\right] \nabla_{X, z} \varphi, \nabla_{X, z} \partial_{\alpha} \varphi\right)_{L^{2}(\mathcal{S})}\right| .
$$

Let us now focus on the second term of the left hand side of (1.14). In order to get an adequate control of this term, we have to write explicitly the commutator $\left[\partial^{\alpha}, \underline{P}\right]$ :

$$
\left[\partial^{\alpha}, \underline{P}\right] \nabla_{X, z} \varphi=\sum_{\substack{\alpha^{\prime}+\alpha^{\prime \prime}=\alpha \\ \alpha^{\prime} \neq 0}} C\left(\left|\alpha^{\prime}\right|,\left|\alpha^{\prime \prime}\right|\right) \partial^{\alpha^{\prime}} \underline{P} \nabla_{X, z} \partial^{\alpha^{\prime \prime}} \varphi
$$

where $C$ is a constant depending only on $\left|\alpha^{\prime}\right|$ and $\left|\alpha^{\prime \prime}\right|$. This leads to the expression

$$
\left(\left[\partial^{\alpha}, \underline{P}\right] \nabla_{X, z} \varphi, \nabla_{X, z} \partial^{\alpha} \varphi\right)_{L^{2}(\mathcal{S})}=\sum_{\substack{\alpha^{\prime}+\alpha^{\prime \prime}=\alpha \\ \alpha^{\prime} \neq 0}} C\left(\left|\alpha^{\prime}\right|,\left|\alpha^{\prime \prime}\right|\right)\left(\partial^{\alpha^{\prime}} \underline{P} \nabla_{X, z} \partial^{\alpha^{\prime \prime}} \varphi, \nabla_{X, z} \partial^{\alpha} \varphi\right)_{L^{2}(\mathcal{S})} .
$$

From now on, we just consider a single term of this sum. Using Proposition 1.3 we derive the explicit expression of $\underline{P}$ and deduce from it the explicit expression of $\partial^{\alpha^{\prime}} \underline{P}$ :

$$
\partial^{\alpha^{\prime}} \underline{P}=\left(\begin{array}{cc}
\left(\partial^{\alpha^{\prime}} \eta-\partial^{\alpha^{\prime}} B\right) P_{d} & P_{d} \partial^{\alpha^{\prime} \mathcal{U}} \\
\left(P_{d} \partial^{\alpha^{\prime} \mathcal{U}}\right)^{T} & \partial^{\alpha^{\prime}}\left(\frac{p_{d+1}+\mathcal{U} \cdot P_{d} \mathcal{U}}{\eta+h_{0}-B}\right)
\end{array}\right)
$$

where $P_{d}$ is the diagonal $(d \times d)$ matrix whose coefficents are $\left(p_{i}\right)_{1 \leq i \leq d}$, and $\mathcal{U}$ the vector defined by $\mathcal{U}=$ $-(z+1) \nabla \eta+z \nabla B$. Using this expression, one easily gets (with $\nabla=\nabla_{X}$ ):

$$
\begin{aligned}
& \left(\partial^{\alpha^{\prime}} \underline{P} \nabla_{X, z} \partial^{\alpha^{\prime \prime}} \varphi, \nabla_{X, z} \partial^{\alpha} \varphi\right)_{L^{2}(\mathcal{S})}=\left(\left(\partial^{\alpha^{\prime}} \eta-\partial^{\alpha^{\prime}} B\right) P_{d} \nabla \partial^{\alpha^{\prime \prime}} \varphi, \nabla \partial^{\alpha} \varphi\right)_{L^{2}(\mathcal{S})}
\end{aligned}
$$

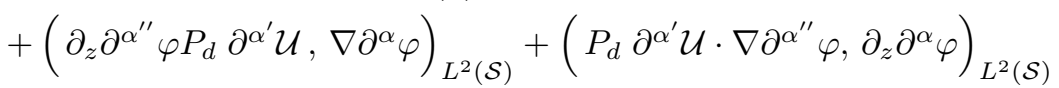

$$
\begin{aligned}
& +\left(\partial^{\alpha^{\prime}}\left(\frac{p_{d+1}+\mathcal{U} \cdot P_{d} \mathcal{U}}{\eta+h_{0}-B}\right) \partial_{z} \partial^{\alpha^{\prime \prime}} \varphi, \partial_{z} \partial^{\alpha} \varphi\right)_{L^{2}(\mathcal{S})} .
\end{aligned}
$$

If we focus on the first term of the right hand side of this equality, we easily get the following intermediate control using Cauchy-Schwarz inequality and the definition of $\|\cdot\|_{\dot{H}^{1}}$ :

$$
\begin{aligned}
\left(\left(\partial^{\alpha^{\prime}} \eta-\partial^{\alpha^{\prime}} B\right) P_{d} \nabla \partial^{\alpha^{\prime \prime}} \varphi, \nabla \partial^{\alpha} \varphi\right)_{L^{2}(\mathcal{S})} & \leq\left(|\eta|_{W\left|\alpha^{\prime}\right|, \infty}+|B|_{W\left|\alpha^{\prime}\right|, \infty}\right)\left\|\sqrt{P_{d}} \nabla \partial^{\alpha^{\prime \prime}} \varphi\right\|_{L^{2}(\mathcal{S})}|| \sqrt{P_{d}} \nabla \partial^{\alpha} \varphi \|_{L^{2}(\mathcal{S})} \\
& \leq\left(|\eta|_{W^{k, \infty}}+|B|_{W^{k, \infty}}\right)\left\|\partial^{\alpha^{\prime \prime}} \varphi\right\|_{\dot{H}^{1}}\left\|\partial^{\alpha} \varphi\right\|_{\dot{H}^{1}} \\
& \leq \frac{\varepsilon^{p}}{\sqrt{p_{d+1}}} C_{k+1}\left(\left\|R^{\varepsilon}\right\|_{H^{k, 0}}+\left|r^{\varepsilon}\right|_{H^{k}}\right)\left\|\partial^{\alpha} \varphi\right\|_{\dot{H}^{1}} .
\end{aligned}
$$

To derive the last inequality, we used the induction hypothesis on $\left\|\partial^{\alpha^{\prime \prime}} \varphi\right\|_{H^{1}}$ since $\left|\alpha^{\prime \prime}\right| \leq m-1$. 
Let us now focus on the second term of the right hand side of (1.15). Using the same arguments as previously and Poincaré inequality, the following controls follow:

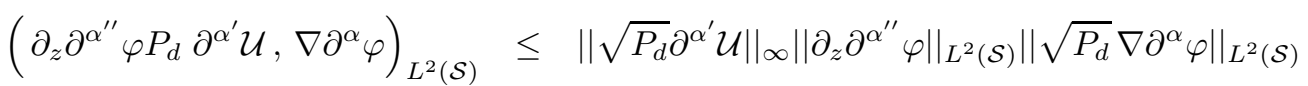

$$
\begin{aligned}
& \leq \sqrt{\frac{\left\|P_{d}\right\|_{\infty}}{p_{d+1}}}\left(|\eta|_{W^{\left|\alpha^{\prime}\right|+1, \infty}}+|B|_{W^{\left|\alpha^{\prime}\right|+1, \infty}}\right)\left\|\partial^{\alpha^{\prime \prime}} \varphi\right\|_{\dot{H}^{1}}\left\|\partial^{\alpha} \varphi\right\|_{\dot{H}^{1}} \\
& \leq \frac{\varepsilon^{p}}{\sqrt{p_{d+1}}} C_{k+1}\left(\left\|R^{\varepsilon}\right\|_{H^{k, 0}}+\left|r^{\varepsilon}\right|_{H^{k}}\right)\left\|\partial^{\alpha} \varphi\right\|_{\dot{H}^{1}},
\end{aligned}
$$

since $\frac{\left\|P_{d}\right\|_{\infty}}{p_{d+1}} \leq \gamma$

The control of the third term of the right hand side of (1.15) comes in the same way:

$$
\left(P_{d} \partial^{\alpha^{\prime}} \mathcal{U} \cdot \nabla \partial^{\alpha^{\prime \prime}} \varphi, \partial_{z} \partial^{\alpha} \varphi\right)_{L^{2}(\mathcal{S})} \leq \frac{\varepsilon^{p}}{\sqrt{p_{d+1}}} C_{k+1}\left(\left\|R^{\varepsilon}\right\|_{H^{k, 0}}+\left|r^{\varepsilon}\right|_{H^{k}}\right)\left\|\partial^{\alpha} \varphi\right\|_{H^{1}} .
$$

The next step consists in controlling the last term of the right hand side of (1.15). We need to do some preliminary work on this term before attempting to bound it adequately. A straightforward computation gives:

$$
\begin{aligned}
\partial^{\alpha^{\prime}}\left(\frac{p_{d+1}+\mathcal{U} \cdot P_{d} \mathcal{U}}{\eta+h_{0}-B}\right)= & \sum_{\substack{\beta_{1}+\beta_{2}=\alpha^{\prime} \\
\beta_{1} \neq 0}} C\left(\left|\beta_{1}\right|,\left|\beta_{2}\right|\right) \partial^{\beta_{1}}\left(\mathcal{U} \cdot P_{d} \mathcal{U}\right) \partial^{\beta_{2}}\left(\frac{1}{\eta+h_{0}-B}\right) \\
& +\mathcal{U} \cdot P_{d} \mathcal{U} \partial^{\alpha^{\prime}}\left(\frac{1}{\eta+h_{0}-B}\right)+p_{d+1} \partial^{\alpha^{\prime}}\left(\frac{1}{\eta+h_{0}-B}\right),
\end{aligned}
$$

from which one deduces:

$$
\begin{aligned}
\left(\partial^{\alpha^{\prime}}\left(\frac{p_{d+1}+\mathcal{U} \cdot P_{d} \mathcal{U}}{\eta+h_{0}-B}\right) \partial_{z} \partial^{\alpha^{\prime \prime}} \varphi, \partial_{z} \partial^{\alpha} \varphi\right)_{L^{2}(\mathcal{S})} \leq & \left\|P_{d}\right\|_{\infty} C_{k+1}\left\|\partial_{z} \partial^{\alpha^{\prime \prime}} \varphi\right\|_{L^{2}(\mathcal{S})}\left\|\partial_{z} \partial^{\alpha} \varphi\right\|_{L^{2}(\mathcal{S})} \\
& +C_{k+1}\left\|\sqrt{p_{d+1}} \partial_{z} \partial^{\alpha^{\prime \prime}} \varphi\right\|_{L^{2}(\mathcal{S})}\left\|\sqrt{p_{d+1}} \partial_{z} \partial^{\alpha} \varphi\right\|_{L^{2}(\mathcal{S})} \\
\leq & \frac{\left\|P_{d}\right\|_{\infty}}{p_{d+1}} C_{k+1}\left\|\partial^{\alpha^{\prime \prime}} \varphi\right\|_{\dot{H}^{1}}\left\|\partial^{\alpha} \varphi\right\|_{\dot{H}^{1}} \\
& +C_{k+1}\left\|\partial^{\alpha^{\prime \prime}} \varphi\right\|_{\dot{H}^{1}}\left\|\partial^{\alpha} \varphi\right\|_{\dot{H}^{1}} \\
\leq & \frac{\varepsilon^{p}}{\sqrt{p_{d+1}}} C_{k+1}\left(\left\|R^{\varepsilon}\right\|_{H^{k, 0}}+\left|r^{\varepsilon}\right|_{H^{k}}\right)\left\|\partial^{\alpha} \varphi\right\|_{\dot{H}^{1}}
\end{aligned}
$$

where we once more used the induction hypothesis.

Gathering the four previous estimates of each term of the right hand side of (1.15) and using the explicit expression of the commutator $\left[\partial^{\alpha}, \underline{P}\right]$ leads to the final estimate of $\left|\left(\left[\partial^{\alpha}, \underline{P}\right] \nabla_{X, z} \varphi, \nabla_{X, z} \partial^{\alpha} \varphi\right)_{L^{2}(\mathcal{S})}\right|$ :

$$
\left|\left(\left[\partial^{\alpha}, \underline{P}\right] \nabla_{X, z} \varphi, \nabla_{X, z} \partial^{\alpha} \varphi\right)_{L^{2}(\mathcal{S})}\right| \leq \frac{\varepsilon^{p}}{\sqrt{p_{d+1}}} C_{k+1}\left(\left\|R^{\varepsilon}\right\|_{H^{k, 0}}+\left|r^{\varepsilon}\right|_{H^{k}}\right)\left\|\partial^{\alpha} \varphi\right\|_{\dot{H}^{1}} .
$$

The last step simply consists in pluging this last estimate into (1.14), which gives:

$$
c_{0}\left(|\eta|_{W^{1, \infty}},|B|_{W^{1, \infty}}\right)\left\|\partial^{\alpha} \varphi\right\|_{\dot{H}^{1}}^{2} \leq \frac{\varepsilon^{p}}{\sqrt{p_{d+1}}}\left\|R^{\varepsilon}\right\|_{H^{k, 0}}\left\|\partial^{\alpha} \varphi\right\|_{\dot{H}^{1}} .
$$


As in the initialization, this last estimate leads to the desired result, which ends the heredity and hence the proof of (1.11).

To conclude this first part of the proof, we use the fact that:

$$
\begin{aligned}
\left\|\partial_{z} \varphi\right\|_{H^{k+1,0}} & \leq C(k+1) \sup _{|\alpha| \leq k+1}\left\|\partial_{z} \partial^{\alpha} \varphi\right\|_{L^{2}(\mathcal{S})} \\
& \leq \frac{C(k+1)}{\sqrt{p_{d+1}}} \sup _{|\alpha| \leq k+1}\left\|\partial^{\alpha} \varphi\right\|_{\dot{H}^{1}},
\end{aligned}
$$

and the estimate (1.11) we just proved to finally get:

$$
\left\|\partial_{z} \varphi\right\|_{H^{k+1,0}} \leq \frac{\varepsilon^{p}}{p_{d+1}} C_{k+2}\left(\left\|R^{\varepsilon}\right\|_{H^{k+1,0}}+\left|r^{\varepsilon}\right|_{H^{k+1}}\right),
$$

which ends the first part of the proof.

2. In this second part, we aim at controlling the quantity $\left\|\partial_{z}^{2} \varphi\right\|_{H^{k, 0}}$. To this end, we prove with a direct method the following estimate:

$$
\left\|\partial_{z}^{2} \varphi\right\|_{H^{k, 0}} \leq \frac{\varepsilon^{p}}{p_{d+1}} C_{k+2}\left(\left\|R^{\varepsilon}\right\|_{H^{k+1,0}}+\left|r^{\varepsilon}\right|_{H^{k+1}}\right)
$$

We first use the equation satisfied by $\varphi$ in order to express $\partial_{z}^{2} \varphi$ in terms of other derivatives of $\varphi$ such as $\nabla \varphi, \partial_{z} \varphi, \nabla \partial_{z} \varphi$ or $\Delta \varphi$ :

$$
\partial_{z}^{2} \varphi=\left(\frac{\eta+h_{0}-B}{p_{d+1}+\mathcal{U} \cdot P_{d} \mathcal{U}}\right)\left[-\nabla_{X, z} \cdot Q \nabla_{X, z} \varphi-\frac{\partial_{z}\left(\mathcal{U} \cdot P_{d} \mathcal{U}\right)}{\eta+h_{0}-B} \partial_{z} \varphi-\varepsilon^{p} R^{\varepsilon}\right]
$$

where $Q=\left(\begin{array}{cc}\left(\eta+h_{0}-B\right) P_{d} & P_{d} \mathcal{U} \\ \left(P_{d} \mathcal{U}\right)^{T} & 0\end{array}\right)$

The following estimates follow (using $\left.\|u\|_{H^{k, 0}} \leq C(k) \sup _{|\alpha| \leq k}\left\|\partial^{\alpha} u\right\|_{L^{2}(\mathcal{S})}\right)$ :

$$
\begin{aligned}
\left\|\partial_{z}^{2} \varphi\right\|_{H^{k, 0}} \leq & \frac{1}{p_{d+1}} C_{k}\left[\left\|\nabla_{X, z} \cdot Q \nabla_{X, z} \varphi\right\|_{H^{k, 0}}+C_{k+1}\left\|P_{d}\right\|_{\infty}\left\|\partial_{z} \varphi\right\|_{H^{k, 0}}+\varepsilon^{p}\left\|R^{\varepsilon}\right\|_{H^{k, 0}}\right] \\
\leq & \frac{1}{p_{d+1}} C_{k+1}\left[C(k) \sup _{|\alpha| \leq k}\left\|\partial^{\alpha}\left(\nabla_{X, z} \cdot Q \nabla_{X, z} \varphi\right)\right\|_{L^{2}(\mathcal{S})}+\frac{\left\|P_{d}\right\|_{\infty}}{\sqrt{p_{d+1}}} C(k) \sup _{|\alpha| \leq k}\left\|\partial^{\alpha} \varphi\right\|_{H^{1}}\right. \\
& \left.+\varepsilon^{p}|| R^{\varepsilon} \|_{H^{k, 0}}\right], \\
\leq & \frac{1}{p_{d+1}} C_{k+1} \sup _{|\alpha| \leq k}\left\|\partial^{\alpha}\left(\nabla_{X, z} \cdot Q \nabla_{X, z} \varphi\right)\right\|_{L^{2}(\mathcal{S})}+f r a c \varepsilon^{p} p_{d+1} \frac{\left\|P_{d}\right\|_{\infty}}{p_{d+1}} C_{k+1}\left(\left\|R^{\varepsilon}\right\|_{H^{k, 0}}+\left|r^{\varepsilon}\right|_{H^{k}}\right) \\
& +\frac{\varepsilon^{p}}{p_{d+1}} C_{k+1}\left\|R^{\varepsilon}\right\|_{H^{k, 0}}, \\
\leq & \frac{1}{p_{d+1}} C_{k+1} \sup _{|\alpha| \leq k}\left\|\partial^{\alpha}\left(\nabla_{X, z} \cdot Q \nabla_{X, z} \varphi\right)\right\|_{L^{2}(\mathcal{S})}+\frac{\varepsilon^{p}}{p_{d+1}} C_{k+1}\left(\left\|R^{\varepsilon}\right\|_{H^{k, 0}}+\left|r^{\varepsilon}\right|_{H^{k}}\right),
\end{aligned}
$$

where we used (1.11) and the fact that $\frac{\left\|P_{d}\right\|_{\infty}}{p_{d+1}} \leq \gamma$.

The last part of the initialization aims at correctly controlling the norm $\left\|\partial^{\alpha}\left(\nabla_{X, z} \cdot Q \nabla_{X, z} \varphi\right)\right\|_{L^{2}(\mathcal{S})}$. The identity

$$
\partial^{\alpha}\left(\nabla_{X, z} \cdot Q \nabla_{X, z} \varphi\right)=\sum_{\alpha^{\prime}+\alpha^{\prime \prime}=\alpha} C\left(\left|\alpha^{\prime}\right|,\left|\alpha^{\prime \prime}\right|\right)\left(\nabla_{X, z} \cdot \partial^{\alpha^{\prime}} Q \nabla_{X, z} \partial^{\alpha^{\prime \prime}} \varphi\right)
$$


and the expression of $Q$ furnish the following estimates:

$$
\begin{aligned}
\left\|\nabla_{X, z} \cdot \partial^{\alpha^{\prime}} Q \nabla_{X, z} \partial^{\alpha^{\prime \prime}} \varphi\right\|_{L^{2}(\mathcal{S})} \leq & C_{\left|\alpha^{\prime}\right|}\left\|\nabla \cdot P_{d} \nabla \partial^{\alpha^{\prime \prime}} \varphi\right\|_{L^{2}(\mathcal{S})} \\
& +C_{\left|\alpha^{\prime}\right|+1}\left\|\sqrt{P_{d}}\right\|_{\infty}\left\|\sqrt{P_{d}} \nabla \partial^{\alpha^{\prime \prime}} \varphi\right\|_{L^{2}(\mathcal{S})} \\
& +C_{\left|\alpha^{\prime}\right|+2}\left\|P_{d}\right\|_{\infty}\left\|\partial_{z} \partial^{\alpha^{\prime \prime}} \varphi\right\|_{L^{2}(\mathcal{S})} \\
& \left.+C_{\left|\alpha^{\prime}\right|+1}\left\|P_{d}\right\|_{\infty}\left\|\partial_{z} \nabla \partial^{\alpha^{\prime \prime}} \varphi\right\|_{L^{2}(\mathcal{S})}\right) \\
\leq & C_{k+2}\left(\left\|\nabla \cdot P_{d} \nabla \partial^{\alpha^{\prime \prime}} \varphi\right\|_{L^{2}(\mathcal{S})}\right. \\
& \left.+\left\|\sqrt{P_{d}}\right\|_{\infty}\left\|\partial^{\alpha^{\prime \prime}} \varphi\right\|_{\dot{H}^{1}}+\frac{\left\|P_{d}\right\|_{\infty}}{\sqrt{p_{d+1}}}\left\|\partial^{\alpha^{\prime \prime}} \varphi\right\|_{\dot{H}^{1}}\right) \\
\leq & C_{k+2}\left(\left\|\nabla \cdot P_{d} \nabla \partial^{\alpha^{\prime \prime}} \varphi\right\|_{L^{2}(\mathcal{S})}+\varepsilon^{p} C_{k+1}\left(\left\|R^{\varepsilon}\right\|_{H^{k, 0}}+\left|r^{\varepsilon}\right|_{H^{k}}\right)\right) .
\end{aligned}
$$

We estimate the term $\left\|\nabla \cdot P_{d} \nabla \partial^{\alpha^{\prime \prime}} \varphi\right\|_{L^{2}(\mathcal{S})}$ using the following technique:

$$
\begin{aligned}
\left\|\nabla \cdot P_{d} \nabla \partial^{\alpha^{\prime \prime}} \varphi\right\|_{L^{2}(\mathcal{S})} & \leq\left\|\sqrt{P_{d}}\right\|_{\infty} \sum_{1 \leq i \leq d}\left\|\sqrt{p_{i}} \partial_{x_{i}}^{2} \partial^{\alpha^{\prime \prime}} \varphi\right\|_{L^{2}(\mathcal{S})} \\
& \leq\left\|\sqrt{P_{d}}\right\|_{\infty} \sum_{1 \leq i \leq d}\left\|\partial_{x_{i}} \partial^{\alpha^{\prime \prime}} \varphi\right\|_{H^{1}} \\
& \leq d\left\|\sqrt{P_{d}}\right\|_{\infty} \sup _{|m|=\left|\alpha^{\prime \prime}\right|+1}\left\|\partial^{m} \varphi\right\|_{\dot{H}^{1}} \\
& \leq d\left\|\sqrt{P_{d}}\right\|_{\infty} \sup _{|m| \leq k+1}\left\|\partial^{m} \varphi\right\|_{\dot{H}^{1}} \\
& \leq \varepsilon^{p} C_{k+2}\left(\left\|R^{\varepsilon}\right\|_{H^{k+1,0}}+\left|r^{\varepsilon}\right|_{H^{k+1}}\right) .
\end{aligned}
$$

We plug this result in (1.19) to obtain

$$
\left\|\nabla_{X, z} \cdot \partial^{\alpha^{\prime}} Q \nabla_{X, z} \partial^{\alpha^{\prime \prime}} \varphi\right\|_{L^{2}(\mathcal{S})} \leq \varepsilon^{p} C_{k+2}\left(\left\|R^{\varepsilon}\right\|_{H^{k+1,0}}+\left|r^{\varepsilon}\right|_{H^{k+1}}\right),
$$

which finally leads to

$$
\left\|\partial^{\alpha}\left(\nabla_{X, z} \cdot Q \nabla_{X, z} \varphi\right)\right\|_{L^{2}(\mathcal{S})} \leq \varepsilon^{p} C_{k+2}\left(\left\|R^{\varepsilon}\right\|_{H^{k+1,0}}+\left|r^{\varepsilon}\right|_{H^{k+1}}\right) .
$$

This way, we get our desired estimate of $\left\|\partial_{z}^{2} \varphi\right\|_{H^{k, 0}}$ :

$$
\left\|\partial_{z}^{2} \varphi\right\|_{H^{k, 0}} \leq \frac{\varepsilon^{p}}{p_{d+1}} C_{k+2}\left(\left\|R^{\varepsilon}\right\|_{H^{k+1,0}}+\left|r^{\varepsilon}\right|_{H^{k+1}}\right) .
$$

Gathering (1.17) and (1.18) in (1.9) ends the proof of the theorem.

\subsection{Application}

We recall that by definition, $Z_{\varepsilon}(\varepsilon \eta, \beta b) f=\partial_{z} u_{\left.\right|_{z=\varepsilon \eta}}$ where $u$ is solution of the boundary value problem

$$
\begin{gathered}
\varepsilon \Delta u+\partial_{z}^{2} u=0 \quad \text { in } \Omega \\
u_{\left.\right|_{z=\varepsilon \eta}}=f, \quad\left(\partial_{z} u-\varepsilon \beta \nabla b \cdot \nabla u\right)_{\left.\right|_{z=-1+\beta b}}=0 .
\end{gathered}
$$


This elliptic problem (1.20)-(1.21) belongs to the class of general elliptic problems (1.2)-(1.3) defined in the previous subsection. The corresponding matrix $P$ is here designed by $P^{\varepsilon}$ :

$$
P^{\varepsilon}=\left(\begin{array}{cc}
\varepsilon I_{d \times d} & 0 \\
0 & 1
\end{array}\right) .
$$

The upper boundary of $\Omega$ is here defined by $\{z=\varepsilon \eta\}$ and the lower one by $\{z=-1+\beta b\}$. We make the additionnal assumption that $\varepsilon$ and $\beta$ are bounded in the following sense: $0<\varepsilon<1$ and there exists a strictly positive constant $\beta_{0}$ such that and $0<\beta<\beta_{0}$. Furthermore, condition (1.1) is here verified thanks to condition (0.1). And finally, we remark that $\left(\frac{p_{i}}{p_{d+1}}\right)_{1 \leq i \leq d}$ are bounded by 1 since $0<\varepsilon<1$. Our goal is here to apply the previous theorem to get asymptotic estimates on $Z_{\varepsilon}(\varepsilon \eta, \beta b)$.

We recall that we are here interested in two differerent regimes depending on the $\beta$ parameter. The first one, namely $\beta=O(\varepsilon)$, refers to the physical case of a bottom with variations of slow amplitude. The second one, namely $\beta=O(1)$, refers on the contrary to variations of high amplitude of the bottom. In order to improve the readability, we take $\beta_{0}=1$ : we thus write $\beta=\varepsilon$ for the first regime and $\beta=1$ for the second one.

\subsubsection{The regime $\beta=\varepsilon$ : small variations of bottom topography}

The boundaries of the domain $\Omega$ are here defined by $\{z=\varepsilon \eta\}$ and $\{z=-1+\varepsilon b\}$ while the matrix $P^{\varepsilon}$ remains as in (1.22). Thanks to Proposition 1.3 we are able to set an equivalent problem to (1.20)-(1.21) defined over the flat band $\mathcal{S}: \underline{u}=u \circ S$ solves the problem:

$$
\begin{gathered}
-\nabla_{X, z} \cdot \underline{P}^{\varepsilon} \nabla_{X, z} \underline{u}=0 \text { in } \mathcal{S}, \\
\underline{u}_{z=0}=f, \quad \partial_{n} \underline{u}_{\mid z=-1}=0,
\end{gathered}
$$

where the matrix $\underline{P}^{\varepsilon}$ is given by

$$
\underline{P}^{\varepsilon}=\left(\begin{array}{cc}
\varepsilon(1+\varepsilon(\eta-b)) I_{d \times d} & -\varepsilon^{2}[(z+1) \nabla \eta-z \nabla b] \\
-\varepsilon^{2}[(z+1) \nabla \eta-z \nabla b]^{T} & \frac{1+\varepsilon^{3}|(z+1) \nabla \eta-z \nabla b|^{2}}{1+\varepsilon(\eta-b)}
\end{array}\right) .
$$

The following result gives a rigourously justified asymptotic expansion of $Z_{\varepsilon}(\varepsilon \eta, \beta b) f$ as $\varepsilon$ goes to 0 :

Proposition 1.7. Let $k \in \mathbb{N}, \eta \in W^{k+2, \infty}\left(\mathbb{R}^{d}\right)$ and $b \in W^{k+2, \infty}\left(\mathbb{R}^{d}\right)$.

Then for all $f$ such that $\nabla f \in H^{k+6}\left(\mathbb{R}^{d}\right)$, we have:

$$
\left|Z_{\varepsilon}(\varepsilon \eta, \beta b) f-\left(\varepsilon Z_{1}+\varepsilon^{2} Z_{2}\right)\right|_{H^{k+1 / 2}} \leq \varepsilon^{3} C_{k+2}|\nabla f|_{H^{k+6}},
$$

with:

$$
\left\{\begin{array}{l}
Z_{1}:=-\Delta f, \\
Z_{2}:=-\frac{1}{3} \Delta^{2} f-(\eta-b) \Delta f+\nabla b \cdot \nabla f .
\end{array}\right.
$$

Proof. To prove this proposition, we use essentially Theorem 1.6 with $p=3$. We know that $\left(\frac{p_{i}}{p_{d+1}}\right)_{1 \leq i \leq d}$ are bounded by 1 . Thus, in order to derive an asymptotic expansion of $Z_{\varepsilon}(\varepsilon \eta, \beta b) f$, we only need to compute an approximate solution $u_{a p p}$ which satisfies the hypothesis of Theorem 1.6 for $p=3$. This approximate solution $u_{a p p}$ can be constructed as in [8] using a classical WKB method, which consists in looking for $u_{\text {app }}$ under the form $u_{\text {app }}=u_{0}+\varepsilon u_{1}+\varepsilon^{2} u_{2}$. We want this function to verify the properties required by Theorem 1.6, that is to say:

$$
\begin{gathered}
-\nabla_{X, z} \cdot \underline{P}^{\varepsilon} \nabla_{X, z} u_{a p p}=\varepsilon^{p} R^{\varepsilon} \quad \text { in } \mathcal{S}, \\
\left.u_{a p p}\right|_{z=0}=f, \quad \partial_{n} u_{\left.a p p\right|_{z=-1}}=\varepsilon^{p} r^{\varepsilon},
\end{gathered}
$$

where $\left(R^{\varepsilon}\right)_{0<\varepsilon<1}$ and $\left(r^{\varepsilon}\right)_{0<\varepsilon<1}$ are bounded independently of $\varepsilon$ respectively in $H^{k+1,0}(\mathcal{S})$ and $H^{k+1}\left(\mathbb{R}^{d}\right)$. 
We decompose the matrix $\underline{P^{\varepsilon}}$ under the form $\underline{P^{\varepsilon}}=P_{0}+\varepsilon P_{1}+\varepsilon^{2} P_{2}+\varepsilon^{3} P_{\varepsilon}$ where $P_{0}, P_{1}, P_{2}$ are independent of $\varepsilon$, and if we plug the desired expression of $u_{a p p}$ into this problem, we get $R^{\varepsilon}=\nabla \cdot T^{\varepsilon}$ and $r^{\varepsilon}=\mathbf{e}_{\mathbf{z}} \cdot T_{\left.\right|_{z=-1}}^{\varepsilon}$ where $T^{\varepsilon}=P_{2} \nabla_{X, z} u_{1}+P_{1} \nabla_{X, z} u_{2}+P_{\varepsilon} \nabla_{X, z}\left(u_{o}+u_{1}+u_{2}\right)$, and the following system of equations and boundary conditions on $u_{0}, u_{1}, u_{2}$ :

$$
\begin{gathered}
\left\{\begin{array}{l}
\partial_{z}^{2} u_{0}=0 \\
\partial_{z}^{2} u_{1}+\left(\Delta-(\eta-b) \partial_{z}^{2}\right) u_{0}=0 \\
\partial_{z}^{2} u_{2}+\left(\Delta-(\eta-b) \partial_{z}^{2}\right) u_{1}+(\eta-b) \Delta u_{0}-2[(z+1) \nabla f-z \nabla b] \\
\nabla \partial_{z} u_{0}-[(z+1) \Delta f-z \Delta b] \cdot \partial_{z} u_{0}-(\eta-b)^{2} \partial_{z}^{2} u_{0}=0
\end{array}\right. \\
\operatorname{with}\left\{\begin{array}{l}
u_{\left.0\right|_{z=0}}=f, \\
u_{\left.i\right|_{z=0}}=0, \quad 1 \leq i \leq 2, \\
\partial_{z} u_{\left.i\right|_{z=-1}}=0, \quad 0 \leq i \leq 1, \\
\partial_{z} u_{\left.2\right|_{z=-1}}-\nabla b \cdot \nabla u_{0 \mid}=-1
\end{array}=0 .\right.
\end{gathered}
$$

We can verify that the following values of $u_{0}, u_{1}, u_{2}$ satisfy the previous equations and boundary conditions:

$$
\begin{aligned}
& u_{0}=f \\
& u_{1}=\left(\frac{1}{2}-\frac{(z+1)^{2}}{2}\right) \Delta f \\
& u_{2}=\left(\frac{(z+1)^{4}}{24}-\frac{(z+1)^{2}}{4}+\frac{5}{24}\right) \Delta^{2} f+\left(1-(z+1)^{2}\right)(\eta-b) \Delta f+z \nabla b \cdot \nabla f .
\end{aligned}
$$

Using these values of $u_{0}, u_{1}$ and $u_{2}$, one can easily obtain the following estimates of $R^{\varepsilon}$ and $r^{\varepsilon}$ :

$$
\left\|R^{\varepsilon}\right\|_{H^{k+1,0}} \leq C_{k+2}|\nabla f|_{H^{k+6}}, \quad\left|r^{\varepsilon}\right|_{H^{k+1}} \leq C_{k+2}|\nabla f|_{H^{k+3}}
$$

Thus $u_{\text {app }}$ satisfies the properties required to apply Theorem 1.6. The last steps of the proof consists in computing $\frac{1}{1+\varepsilon(\eta-b)} \partial_{z} u_{\left.a p p\right|_{z=0}}$ using the explicit expression of $u_{a p p}$ previously determined, and then apply Theorem 1.6. An easy Taylor expansion of $\frac{1}{1+\varepsilon(\eta-b)} \partial_{z} u_{\left.a p p\right|_{z=0}}$ then yields the result.

Remark 1.8. The method developped here to get and prove our asymptotic expansion is improved compared to the one developped in [8] since we do not need here to compute the term $u_{3}$.

Remark 1.9. If we take $b=0-i . e$. if we consider a flat bottom - , we of course get the same expansion as the ones proved in [8].

\subsubsection{The regime $\beta=1$ : strong variations of bottom topography}

The boundaries of the domain $\Omega$ are here defined by $\{z=\varepsilon \eta\}$ and $\{z=-1+b\}$. Using again Proposition 1.3, we set an equivalent problem to (1.20)-(1.21) defined over the flat band $\mathcal{S}$ : this new problem is the same as the one defined in the first regime, at the exception of the matrix $\underline{P}^{\varepsilon}$ which is now given by

$$
\underline{P}^{\varepsilon}=\left(\begin{array}{cc}
\varepsilon(1+\varepsilon \eta-b) I_{d \times d} & -\varepsilon[\varepsilon(z+1) \nabla \eta-z \nabla b] \\
-\varepsilon[\varepsilon(z+1) \nabla \eta-z \nabla b]^{T} & \frac{1+\varepsilon|\varepsilon(z+1) \nabla \eta-z \nabla b|^{2}}{1+\varepsilon \eta-b}
\end{array}\right) .
$$

As in the first regime we give a rigourously justified asymptotic expansion of $Z_{\varepsilon}(\varepsilon \eta, \beta b) f$ in the present regime. 
Proposition 1.10. Let $k \in \mathbb{N}, \eta \in W^{k+2, \infty}\left(\mathbb{R}^{d}\right)$ and $b \in W^{k+2, \infty}\left(\mathbb{R}^{d}\right)$.

Then for all $f$ such that $\nabla f \in H^{k+6}\left(\mathbb{R}^{d}\right)$, we have:

$$
\left|Z_{\varepsilon}(\varepsilon \eta, \beta b) f-\left(\varepsilon Z_{1}+\varepsilon^{2} Z_{2}\right)\right|_{H^{k+1 / 2}} \leq \varepsilon^{3} C_{k+2}|\nabla f|_{H^{k+6}},
$$

with:

$$
\left\{\begin{array}{l}
Z_{1}:=-\nabla \cdot((1-b) \nabla f), \\
Z_{2}:=\frac{1}{2} \nabla \cdot\left(\frac{1}{3}(1-b)^{3} \nabla \Delta f-(1-b)^{2} \nabla \nabla \cdot((1-b) \nabla f)\right)-\eta \Delta f .
\end{array}\right.
$$

Proof. The proof of this proposition follows exactly the same steps as the proof of Proposition 1.7. The following values of $u_{0}, u_{1}, u_{2}$ are found:

$$
\begin{aligned}
u_{0}= & f \\
u_{1}= & \frac{(1-b)^{2}}{2}\left(1-(z+1)^{2}\right) \Delta f+z(1-b) \nabla b \cdot \nabla f, \\
u_{2}= & \frac{(1-b)^{4}}{24} \Delta^{2} f z^{4}+f r a c(1-b)^{3} 6 \Delta \nabla \cdot((1-b) \nabla f) z^{3}-(1-b) \eta \Delta f z^{2} \\
& +\left[\frac{(1-b)}{2} \nabla \cdot\left(\frac{(1-b)^{3}}{3} \nabla \Delta f-(1-b)^{2} \nabla \nabla \cdot((1-b) \nabla f)\right)\right. \\
& -\eta(2(1-b) \Delta f+\nabla b \cdot \nabla f)] z .
\end{aligned}
$$

The error bound is derived in the same way and the previous values lead to the result.

Remark 1.11. By formally taking $b=\varepsilon b$, we recover the result of Proposition 1.7.

\subsection{Derivation of Boussinesq-like models for uneven bottoms}

We recall the Zakharov formulation of the water waves equations, from which we intend to derive new systems using the previous results:

$$
\left(S_{0}\right)\left\{\begin{array}{l}
\partial_{t} \psi-\varepsilon \partial_{t} \eta Z_{\varepsilon}(\varepsilon \eta, \beta b) \psi+\frac{1}{2}\left[\varepsilon\left|\nabla \psi-\varepsilon \nabla \eta Z_{\varepsilon}(\varepsilon \eta, \beta b) \psi\right|^{2}+\left|Z_{\varepsilon}(\varepsilon \eta, \beta b) \psi\right|^{2}\right]+\eta=0, \\
\partial_{t} \eta+\varepsilon \nabla \eta \cdot\left[\nabla \psi-\varepsilon \nabla \eta Z_{\varepsilon}(\varepsilon \eta, \beta b) \psi\right]=\frac{1}{\varepsilon} Z_{\varepsilon}(\varepsilon \eta, \beta b) \psi .
\end{array}\right.
$$

As in [8], we introduce the notion of consistency.

Definition 1.12. Let $\sigma, s \in \mathbb{R}, \varepsilon_{0}>0, T>0$ and let $\left(V^{\varepsilon}, \eta^{\varepsilon}\right)_{0<\varepsilon<\varepsilon_{0}}$ be bounded in $W^{1, \infty}\left(\left[0, \frac{T}{\varepsilon}\right] ; H^{\sigma}\left(\mathbb{R}^{d}\right)^{d+1}\right)$ independently of $\varepsilon$. This family is called consistent (at order $s$ ) with a system $(S)$ if it is solution of $(S)$ with a residual of order $\varepsilon^{2}$ in $L^{\infty}\left(\left[0, \frac{T}{\varepsilon}\right] ; H^{s}\left(\mathbb{R}^{d}\right)^{d+1}\right)$.

We are now able to state the following results which show the consistency of two Boussinesq-like systems with the system $\left(S_{0}\right)$. We introduce here the quantity $h=1-b$ which corresponds to the non-dimensional still water depth. From now on, this quantity is considered as a topography term since it only depends on $b$.

Proposition 1.13 (small variations regime $\beta=\varepsilon$ ). Let $T>0, s \geq 0, \sigma \geq s$ and $\left(\psi^{\varepsilon}, \eta^{\varepsilon}\right)_{0<\varepsilon<\varepsilon_{0}}$ be a family of solutions of $(0.7)$ such that $\left(\nabla \psi^{\varepsilon}, \eta^{\varepsilon}\right)_{0<\varepsilon<\varepsilon_{0}}$ is bounded with respect to $\varepsilon$ in $W^{1, \infty}\left(\left[0, \frac{T}{\varepsilon}\right] ; H^{\sigma}\left(\mathbb{R}^{d}\right)^{d+1}\right)$ with $\sigma$ 
large enough. We define $V^{\varepsilon}:=\nabla \psi^{\varepsilon}$. Then the family $\left(V^{\varepsilon}, \eta\right)_{0<\varepsilon<\varepsilon_{0}}$ is consistent with the following system:

$$
\left(\mathcal{B}_{1}\right)\left\{\begin{array}{l}
\partial_{t} V+\nabla \eta+\frac{\varepsilon}{2} \nabla|V|^{2}=0, \\
\partial_{t} \eta+\nabla \cdot V+\varepsilon\left[\nabla \cdot((\eta-b) V)+\frac{1}{3} \Delta \nabla \cdot V\right]=0 .
\end{array}\right.
$$

Proof. This is clear thanks to the asymptotic expansion of the operator $Z_{\varepsilon}(\varepsilon \eta, \beta b)$ : plugging this in system (0.7), neglecting the terms of order $O\left(\varepsilon^{2}\right)$, and taking the gradient yields the result.

Proposition 1.14 (strong variations regime $\beta=1$ ). Let $T>0, s \geq 0, \sigma \geq s$ and $\left(\psi^{\varepsilon}, \eta^{\varepsilon}\right)_{0<\varepsilon<\varepsilon_{0}}$ be a family of solutions of $(0.7)$ such that $\left(\nabla \psi^{\varepsilon}, \eta^{\varepsilon}\right)_{0<\varepsilon<\varepsilon_{0}}$ is bounded with respect to $\varepsilon$ in $W^{1, \infty}\left(\left[0, \frac{T}{\varepsilon}\right] ; H^{\sigma}\left(\mathbb{R}^{d}\right)^{d+1}\right)$ with $\sigma$ large enough. We define $V^{\varepsilon}:=\nabla \psi^{\varepsilon}$. Then the family $\left(V^{\varepsilon}, \eta\right)_{0<\varepsilon<\varepsilon_{0}}$ is consistent with the following system (with $h=1-b$ ):

$$
\left(\mathcal{B}_{2}\right)\left\{\begin{array}{l}
\partial_{t} V+\nabla \eta+\frac{\varepsilon}{2} \nabla|V|^{2}=0, \\
\partial_{t} \eta+\nabla \cdot(h V)+\varepsilon\left[\nabla \cdot(\eta V)-\frac{1}{2} \nabla \cdot\left(\frac{h^{3}}{3} \nabla \nabla \cdot V-h^{2} \nabla \nabla \cdot(h V)\right)\right]=0 .
\end{array}\right.
$$

These results close the first section. In the next sections, we separately study the two regimes $\beta=\varepsilon$ and $\beta=1$. The Boussinesq-like systems $\left(\mathcal{B}_{1}\right)$ and $\left(\mathcal{B}_{2}\right)$ are respectively the starting points of these studies.

\section{The REgime OF SMALL TOPOGRAPHY VARIATIONS}

We recall the previously derived Boussinesq-like system $\left(\mathcal{B}_{1}\right)$ on which we base our analysis:

$$
\left(\mathcal{B}_{1}\right)\left\{\begin{array}{l}
\partial_{t} V+\nabla \eta+\frac{\varepsilon}{2} \nabla|V|^{2}=0, \\
\partial_{t} \eta+\nabla \cdot V+\varepsilon\left[\nabla \cdot((\eta-b) V)+\frac{1}{3} \Delta \nabla \cdot V\right]=0 .
\end{array}\right.
$$

We now follow the method put forward in [7] and [8] to derive equivalent systems to $\left(\mathcal{B}_{1}\right)$ in the meaning of consistency. The rigourous justifications of the derivation of these systems is adressed in Section 2 in [8].

\subsection{A first class of equivalent systems}

As in [7] (for the 1D case) and in [8], we define:

$$
V_{\theta}=\left(1+\frac{\varepsilon}{2}\left(1-\theta^{2}\right) \Delta\right) V
$$

which corresponds to the approximation at the order $\varepsilon^{2}$ of the horizontal component of the velocity field at height $-1+\theta$ for $\theta \in[0,1]$. If we remark that $V_{\theta}=\left(1+\frac{\varepsilon}{2}\left(\theta^{2}-1\right) \Delta\right)^{-1} V+O\left(\varepsilon^{2}\right)$, the expression of $V_{\theta}$ in terms of $V$ comes in the following way by supposing $V$ regular enough:

$$
V=\left(1+\frac{\varepsilon}{2}\left(\theta^{2}-1\right) \Delta\right) V_{\theta}+O\left(\varepsilon^{2}\right)
$$

where $O\left(\varepsilon^{2}\right)$ is to be taken in the $L^{\infty}\left(\left[0, \frac{T}{\varepsilon}\right], H^{s}\left(\mathbb{R}^{d}\right)\right)$ norm. 
Plugging this relation into the system $\left(\mathcal{B}_{1}\right)$ leads to:

$$
\left\{\begin{array}{l}
\partial_{t} V_{\theta}+\nabla \eta+\frac{\varepsilon}{2}\left(\nabla|V|^{2}+\left(\theta^{2}-1\right) \Delta \partial_{t} V_{\theta}\right)=O\left(\varepsilon^{2}\right) \\
\partial_{t} \eta+\nabla \cdot V_{\theta}+\varepsilon\left[\nabla \cdot((\eta-b) V)+\left(\frac{\theta^{2}}{2}-\frac{1}{6}\right) \Delta \nabla \cdot V_{\theta}\right]=O(\varepsilon)^{2}
\end{array}\right.
$$

At this point we use the classical BBM trick which consists in using the equations to write:

$$
\begin{aligned}
\partial_{t} V_{\theta} & =-\nabla \eta+O(\varepsilon)=(1-\mu) \partial_{t} V_{\theta}-\mu \nabla \eta+O(\varepsilon), \\
\nabla \cdot V_{\theta} & =-\partial_{t} \eta+O(\varepsilon)=\lambda \nabla \cdot V_{\theta}-(1-\lambda) \partial_{t} \eta+O(\varepsilon),
\end{aligned}
$$

where $\lambda$ and $\mu$ are two real parameters.

We plug these relations into the dispersive terms of the last system to get:

$$
\left\{\begin{array}{l}
\partial_{t} V_{\theta}+\nabla \eta+\frac{\varepsilon}{2}\left[\nabla\left|V_{\theta}\right|^{2}-\mu\left(\theta^{2}-1\right) \Delta \nabla \eta-(\mu-1)\left(\theta^{2}-1\right) \Delta \partial_{t} V_{\theta}\right]=O\left(\varepsilon^{2}\right), \\
\partial_{t} \eta+\nabla \cdot V_{\theta}+\varepsilon\left[\nabla \cdot\left((\eta-b) V_{\theta}\right)+\lambda\left(\frac{\theta^{2}}{2}-\frac{1}{6}\right) \Delta \nabla \cdot V_{\theta}-(1-\lambda)\left(\frac{\theta^{2}}{2}-\frac{1}{6}\right) \Delta \partial_{t} \eta\right]=O\left(\varepsilon^{2}\right) .
\end{array}\right.
$$

We then introduce the class $\mathcal{S}$ of all the systems of the previous form: these systems are denoted by $S_{\theta, \lambda, \mu}$ and can be rewritten in compact form:

$$
\left(S_{\theta, \lambda, \mu}^{1}\right)\left\{\begin{array}{l}
\left(1-\varepsilon a_{2} \Delta\right) \partial_{t} V_{\theta}+\nabla \eta+\varepsilon\left[\frac{1}{2} \nabla\left|V_{\theta}\right|^{2}+a_{1} \Delta \nabla \eta\right]=0 \\
\left(1-\varepsilon a_{4} \Delta\right) \partial_{t} \eta+\nabla \cdot V_{\theta}+\varepsilon\left[\nabla \cdot\left((\eta-b) V_{\theta}\right)+a_{3} \Delta \nabla \cdot V_{\theta}\right]=0
\end{array}\right.
$$

with

$$
\begin{aligned}
& a_{1}=-\mu \frac{\theta^{2}-1}{2}, \quad a_{2}=(\mu-1) \frac{\theta^{2}-1}{2}, \\
& a_{3}=\lambda\left(\frac{\theta^{2}}{2}-\frac{1}{6}\right), \quad a_{4}=(1-\lambda)\left(\frac{\theta^{2}}{2}-\frac{1}{6}\right) .
\end{aligned}
$$

On this class $\mathcal{S}$, the previous computations give us the following two consistency results:

Proposition 2.1. Let $\theta \in[0,1]$ and $\left(\psi^{\varepsilon}, \eta^{\varepsilon}\right)_{0<\varepsilon<\varepsilon_{0}}$ be a family of solutions of $(0.7)$ such that $\left(\nabla \psi^{\varepsilon}, \eta^{\varepsilon}\right)_{0<\varepsilon<\varepsilon_{0}}$ is bounded with respect to $\varepsilon$ in $W^{1, \infty}\left(\left[0, \frac{T}{\varepsilon}\right] ; H^{\sigma}\left(\mathbb{R}^{d}\right)^{d+1}\right)$ with $\sigma$ large enough. We define $V^{\varepsilon}=\nabla \psi^{\varepsilon}$ and $V_{\theta}^{\varepsilon}=\left(1+\frac{\varepsilon}{2}\left(1-\theta^{2}\right) \Delta\right) V^{\varepsilon}$. Then for all $(\lambda, \mu) \in \mathbb{R}^{2}$, the family $\left(V_{\theta}^{\varepsilon}, \eta^{\varepsilon}\right)_{0<\varepsilon<\varepsilon_{0}}$ is consistent with the system $\left(S_{\theta, \lambda, \mu}^{1}\right)$.

Proof. We saw in the previous section that if $\left(\psi^{\varepsilon}, \eta^{\varepsilon}\right)_{0<\varepsilon<\varepsilon_{0}}$ is a family of solutions of $(0.7)$, then the family $\left(\nabla \psi^{\varepsilon}, \eta^{\varepsilon}\right)_{0<\varepsilon<\varepsilon_{0}}$ is consistent with the system $\left(\mathcal{B}_{1}\right)$. Thanks to the previous computations, and since the choice of the parameters $(\lambda, \mu)$ is totally free, it is clear that $\left(V_{\theta}^{\varepsilon}, \eta^{\varepsilon}\right)_{0<\varepsilon<\varepsilon_{0}}$ is consistent with any system $\left(S_{\theta, \lambda, \mu}^{1}\right)$.

Proposition 2.2. Up to a change of variables, all the systems belonging to the class $\mathcal{S}$ are equivalent in the meaning of consistency.

Proof. Let $(\theta, \lambda, \mu) \in[0,1] \times \mathbb{R}^{2}$ and $\left(V_{\theta}^{\varepsilon}, \eta^{\varepsilon}\right)_{0<\varepsilon<\varepsilon_{0}}$ a family consistent with $\left(S_{\theta, \lambda, \mu}^{1}\right)$. We then define, for $\theta_{1} \in[0,1]$

$$
V_{\theta_{1}}^{\varepsilon}=\left(1+\frac{\varepsilon}{2}\left(1-\theta_{1}^{2}\right) \Delta\right)\left(1-\frac{\varepsilon}{2}\left(1-\theta^{2}\right) \Delta\right) V_{\theta}^{\varepsilon}
$$


using the fact that $\left(1-\frac{\varepsilon}{2}\left(1-\theta^{2}\right) \Delta\right) V_{\theta}^{\varepsilon}=V^{\varepsilon}+O\left(\varepsilon^{2}\right)$ and the previous proposition, we easily deduce that the family $\left(V_{\theta_{1}}^{\varepsilon}, \eta^{\varepsilon}\right)_{0<\varepsilon<\varepsilon_{0}}$ is consistent with any system $\left(S_{\theta_{1}, \lambda_{1}, \mu_{1}}^{1}\right)$ for any $\left(\lambda_{1}, \mu_{1}\right) \in \mathbb{R}^{2}$.

\section{Remarks 2.3.}

- By taking $\theta=1, \lambda=1, \mu=0$, we remark that the previously derived Boussinesq-like system $\mathcal{B}_{1}$ is actually a member of the class $\mathcal{S}$.

- By taking $\lambda=\mu=1 / 2$ and $\theta^{2}=2 / 3$, we get $a_{1}=a_{2}=a_{3}=a_{4}=\frac{1}{12}$, so that the dispersive part of the correponding system $\left(S_{\theta, \lambda, \mu}^{1}\right)$ is symmetric. However, the nonlinear terms, that are not affected by the choice of $\theta, \lambda, \mu$, are not symmetric: this problem is adressed in the next section.

- In [10], Chen formally studied in $1 \mathrm{D}$ the case of slowly variating bottoms and derived the same class of systems at the exception that she considered time-dependent bottoms: her systems thus contain additionnal time derivative terms on the bottom that does not appear here but could be easily obtained for a time dependent bottom.

\subsection{A second class of equivalent systems}

Adapting the nonlinear change of variables of [8] to the present case of varying depth, we introduce $\tilde{V}$ :

$$
\tilde{V}=\left(1+\frac{\varepsilon}{2}(\eta-b)\right) V
$$

This nonlinear change of variable symetrizes the nonlinear part of the equations.

This change of variables only affects the nonlinear terms and not the dispersive terms. If $\left(V^{\varepsilon}, \eta^{\varepsilon}\right)_{0<\varepsilon<\varepsilon_{0}}$ is consistent with a system $\left(S_{\theta, \lambda, \mu}^{1}\right)$ of the class $\mathcal{S}$, then $\tilde{V}^{\varepsilon}=\left(1+\frac{\varepsilon}{2} \eta^{\varepsilon}\right) V^{\varepsilon}$ and $\eta^{\varepsilon}$ satisfy the following equations:

$$
\left\{\begin{array}{l}
\left(1-\varepsilon a_{2} \Delta\right) \partial_{t} \tilde{V}^{\varepsilon}+\nabla \eta^{\varepsilon}+\varepsilon\left[\frac{1}{4} \nabla\left|\eta^{\varepsilon}\right|^{2}+\frac{1}{2} \nabla\left|\tilde{V}^{\varepsilon}\right|^{2}+\frac{1}{2} \tilde{V}^{\varepsilon} \nabla \cdot \tilde{V}^{\varepsilon}-\frac{1}{2} b \nabla \eta^{\varepsilon}+a_{1} \Delta \nabla \eta^{\varepsilon}\right]=O\left(\varepsilon^{2}\right), \\
\left(1-\varepsilon a_{4} \Delta\right) \partial_{t} \eta^{\varepsilon}+\nabla \cdot \tilde{V}^{\varepsilon}+\varepsilon\left[\frac{1}{2} \nabla \cdot\left(\left(\eta^{\varepsilon}-b\right) \tilde{V}^{\varepsilon}\right)+a_{3} \Delta \nabla \cdot \tilde{V}^{\varepsilon}\right]=O\left(\varepsilon^{2}\right) .
\end{array}\right.
$$

As observed in [8], if we consider a two-dimensional domain, that is to say $d=1$, the nonlinear terms are actually symmetric. But this is not the case in a three-dimensional domain. However we can deal with this problem for $d=2$ using the following remark coming from [18]:

$$
\frac{1}{2} \nabla\left|\tilde{V}^{\varepsilon}\right|^{2}=\frac{1}{4} \nabla\left|\tilde{V}^{\varepsilon}\right|^{2}+\frac{1}{2}\left(\tilde{V}^{\varepsilon} \cdot \nabla\right) \tilde{V}^{\varepsilon}+\frac{1}{2} \tilde{V}^{\varepsilon} \wedge\left(\nabla \times \tilde{V}^{\varepsilon}\right)
$$

Assuming that $\nabla \times \tilde{V}^{\varepsilon}=O(\varepsilon)$, one formally derives the following system:

$$
\left\{\begin{aligned}
\left(1-\varepsilon a_{2} \Delta\right) \partial_{t} \tilde{V}^{\varepsilon}+\nabla \eta^{\varepsilon}+\varepsilon & {\left[\frac{1}{4} \nabla\left|\eta^{\varepsilon}\right|^{2}+\frac{1}{4} \nabla\left|\tilde{V}^{\varepsilon}\right|^{2}+\frac{1}{2}\left(\tilde{V}^{\varepsilon} \cdot \nabla\right) \tilde{V}^{\varepsilon}\right.} \\
& \left.+\frac{1}{2} \tilde{V}^{\varepsilon} \nabla \cdot \tilde{V}^{\varepsilon}-\frac{1}{2} b \nabla \eta^{\varepsilon}+a_{1} \Delta \nabla \eta^{\varepsilon}\right]=O\left(\varepsilon^{2}\right), \\
\left(1-\varepsilon a_{4} \Delta\right) \partial_{t} \eta^{\varepsilon}+\nabla \cdot \tilde{V}^{\varepsilon}+\varepsilon & {\left[\frac{1}{2} \nabla \cdot\left(\left(\eta^{\varepsilon}-b\right) \tilde{V}^{\varepsilon}\right)+a_{3} \Delta \nabla \cdot \tilde{V}^{\varepsilon}\right]=O\left(\varepsilon^{2}\right) . }
\end{aligned}\right.
$$

The nonlinear terms of the previous system are now symmetric regardless of the dimension. This previous computations are summed up in the following proposition: 
Proposition 2.4. Let $\left(V^{\varepsilon}, \eta^{\varepsilon}\right)_{0<\varepsilon<\varepsilon_{0}}$ be a family consistent with a system $\left(S_{\theta, \lambda, \mu}^{1}\right)$ and $\tilde{V}^{\varepsilon}=\left(1+\frac{\varepsilon}{2} \eta^{\varepsilon}\right) V^{\varepsilon}$. If $\nabla \times \tilde{V}^{\varepsilon}=O(\varepsilon)$, then the family $\left(\tilde{V}^{\varepsilon}, \eta^{\varepsilon}\right)_{0<\varepsilon<\varepsilon_{0}}$ is consistent with the following system:

$$
\left(T_{\theta, \lambda, \mu}^{1}\right)\left\{\begin{array}{l}
\left(1-\varepsilon a_{2} \Delta\right) \partial_{t} V+\nabla \eta+\varepsilon\left[\frac{1}{4} \nabla|\eta|^{2}+\frac{1}{4} \nabla|V|^{2}+\frac{1}{2}(V \cdot \nabla) V+\frac{1}{2} V \nabla \cdot V-\frac{1}{2} b \nabla \eta+a_{1} \Delta \nabla \eta\right]=0, \\
\left(1-\varepsilon a_{4} \Delta\right) \partial_{t} \eta+\nabla \cdot V+\varepsilon\left[\frac{1}{2} \nabla \cdot((\eta-b) V)+a_{3} \Delta \nabla \cdot V\right]=0 .
\end{array}\right.
$$

We introduce the class $\mathcal{T}$ composed with the systems of the form $\left(T_{\theta, \lambda, \mu}^{1}\right)$ for any $(\theta, \lambda, \mu) \in[0,1] \times \mathbb{R}^{2}$. Using this result, we prove the following proposition:

Proposition 2.5. Let $\theta \in[0,1]$ and $\left(\psi^{\varepsilon}, \eta^{\varepsilon}\right)_{0<\varepsilon<\varepsilon_{0}}$ be a family of solutions of $(0.7)$ such that $\left(\nabla \psi^{\varepsilon}, \eta^{\varepsilon}\right)_{0<\varepsilon<\varepsilon_{0}}$ is bounded with respect to $\varepsilon$ in $W^{1, \infty}\left(\left[0, \frac{T}{\varepsilon}\right] ; H^{\sigma}\left(\mathbb{R}^{d}\right)^{d+1}\right)$ with $\sigma$ large enough.

We define $\tilde{V}^{\varepsilon}=\left(1+\frac{\varepsilon}{2}(\eta-b)\right)\left(1+\frac{\varepsilon}{2}\left(1-\theta^{2}\right) \Delta\right) \nabla \psi^{\varepsilon}$. Then for all $(\lambda, \mu) \in \mathbb{R}^{2}$, the family $\left(\tilde{V}^{\varepsilon}, \eta^{\varepsilon}\right)_{0<\varepsilon<\varepsilon_{0}}$ is consistent with the system $\left(T_{\theta, \lambda, \mu}^{1}\right)$.

Proof. Thanks to Proposition 2.1, the family $\left(V_{\theta}^{\varepsilon}, \eta^{\varepsilon}\right)_{0<\varepsilon<\varepsilon_{0}}$ is consistent with the system $\left(S_{\theta, \lambda, \mu}^{1}\right)$ for all $(\lambda, \mu) \in$ $\mathbb{R}^{2}$, where $V_{\theta}^{\varepsilon}=\left(1+\frac{\varepsilon}{2}\left(1-\theta^{2}\right) \Delta\right) V^{\varepsilon}$ and $V^{\varepsilon}=\nabla \psi^{\varepsilon}$. We then use the following remark: by hypothesis, the 3D velocity field $V_{\text {euler }}^{\varepsilon}$ of the fluid is irrotationnal, thus using the introduction notations: $\nabla \times \nabla \phi^{\varepsilon}=\nabla \times \nabla \psi^{\varepsilon}=0$ and hence $\nabla \times V^{\varepsilon}=0$. We easily deduce that $\nabla \times V_{\theta}^{\varepsilon}=O(\varepsilon)$ and $\nabla \times \tilde{V}^{\varepsilon}=O(\varepsilon)$. Applying the previous proposition yields the result.

\subsection{A new class of completely symmetric systems}

We remarked in the first section that there exists values of $(\theta, \lambda, \mu)$ such that the dispersive terms are symmetric. Consequently, the corresponding system $\left(T_{\theta, \lambda, \mu}^{1}\right)$ of the class $\mathcal{T}$ is completely symmetric since both its dispersive terms and nonlinear terms are symmetric. We thus introduce the non-empty subclass of $\mathcal{T}$ denoted by $\Sigma$ composed with the systems of the form $\left(T_{\theta, \lambda, \mu}^{1}\right)$ for which we have $a_{1}=a_{3}, a_{2} \geq 0, a_{4} \geq 0$. The first condition $a_{1}=a_{3}$ symetrizes the nonlinear terms and the last ones $a_{2} \geq 0, a_{4} \geq 0$ ensure the well-posedness of these completely symmetric systems. Indeed, one of the great advantages of these systems belonging to $\Sigma$ is that we have a well-posedness over a large time scale:

Proposition 2.6. Let $s>\frac{d}{2}+1$ and $(\theta, \lambda, \mu)$ be such that the system $\left(T_{\theta, \lambda, \mu}^{1}\right)$ belongs to the class $\Sigma$.

Then for all $\left(V_{0}, \eta_{0}\right) \in H^{s}\left(\mathbb{R}^{d}\right)^{d+1}$, there exists a time $T_{0} \geq 0$ independent of $\varepsilon$ and a unique solution $(V, \eta) \in C\left(\left[0, \frac{T_{0}}{\varepsilon}\right] ; H^{s}\left(\mathbb{R}^{d}\right)^{d+1}\right) \cap C^{1}\left(\left[0, \frac{T_{0}}{\varepsilon}\right] ; H^{s-3}\left(\mathbb{R}^{d}\right)^{d+1}\right)$ to the system $\left(T_{\theta, \lambda, \mu}^{1}\right)$ such that $(V, \eta)_{\mid t=0}=\left(V_{0}, \eta_{0}\right)$.

Furthermore, this unique solution is bounded independently of $\varepsilon$ in the following sense: there exists a constant $C_{0}$ independent of $\varepsilon$ such that for all $k$ verifying $s-3 k>\frac{d}{2}+1$, we have:

$$
|(V, \eta)|_{W^{k, \infty}\left(\left[0, \frac{T_{0}}{\varepsilon}\right] ; H^{s-3 k}\left(\mathbb{R}^{d}\right)^{d+1}\right)} \leq C_{0} .
$$

Proof. This theorem is a very classical result on hyperbolic symmetric quasilinear systems, and we omit the proof here.

As in [8], we are now able to rigorously construct approximate solutions to the water waves problem from the solutions of any of these symmetric systems.

More precisely, let us consider a solution $\left(\psi^{\varepsilon}, \eta^{\varepsilon}\right)$ to the initial system $(0.7)$ with initial data $\left(\psi_{0}^{\varepsilon}, \eta_{0}^{\varepsilon}\right)$ such that $\left(\nabla \psi_{0}^{\varepsilon}, \eta_{0}^{\varepsilon}\right) \in H^{s}\left(\mathbb{R}^{d}\right)^{d+1}$ for a suitably large value of $s$. We define $V^{\varepsilon}=\nabla \psi^{\varepsilon}$ and $V_{0}^{\varepsilon}=\nabla \psi_{0}^{\varepsilon}$. From this solution of the water waves problem, we construct an approximate solution as follows: 
- We first construct what we call here approximate initial data, by applying the two successive changes of variable on the data $\left(V_{0}^{\varepsilon}, \eta_{0}^{\varepsilon}\right)$ :

$$
\left\{\begin{array}{l}
V_{\Sigma, 0}^{\varepsilon}=\left(1+\frac{\varepsilon}{2}\left(\eta_{0}^{\varepsilon}-b\right)\right)\left(1+\frac{\varepsilon}{2}\left(1-\theta^{2}\right) \Delta\right) V_{0}^{\varepsilon}, \\
\eta_{\Sigma, 0}^{\varepsilon}=\eta_{0}^{\varepsilon} .
\end{array}\right.
$$

- We then choose the parameters $(\theta, \lambda, \mu) \in[0,1] \times \mathbb{R}^{2}$ such that the system $\left(T_{\theta, \lambda, \mu}^{1}\right)$ belongs to the class $\Sigma$ of completely symmetric systems (this choice is always possible as we saw previously). Using Proposition 2.6, we know that there exists a unique solution to this system with initial data $\left(V_{\Sigma, 0}^{\varepsilon}, \eta_{\Sigma, 0}^{\varepsilon}\right)$ : we denote this solution by $\left(V_{\Sigma}^{\varepsilon}, \eta_{\Sigma}^{\varepsilon}\right)$.

- From this exact solution of the symmetric system $\left(T_{\theta, \lambda, \mu}^{1}\right)$, we finally construct an approximate solution of the water waves problem by successively and approximatively inverting the two changes of variable as shown below:

$$
\left\{\begin{array}{l}
V_{a p p}^{\varepsilon}=\left(1-\frac{\varepsilon}{2}\left(1-\theta^{2}\right) \Delta\right)\left[\left(1-\frac{\varepsilon}{2}\left(\eta_{\Sigma}^{\varepsilon}-b\right)\right) V_{\Sigma}^{\varepsilon}\right] \\
\eta_{a p p}^{\varepsilon}=\eta_{\Sigma}^{\varepsilon} .
\end{array}\right.
$$

This formal construction of an approximate solution founds its mathematical justification in the following theorem which is the last result of this section.

Theorem 2.7. Let $T_{1} \geq 0, s \geq \frac{d}{2}+1, \sigma \geq s+3$ and $\left(\nabla \psi_{0}^{\varepsilon}, \eta_{0}^{\varepsilon}\right)$ be in $H^{\sigma}\left(\mathbb{R}^{d}\right)^{d+1}$. Let $\left(\psi^{\varepsilon}, \eta^{\varepsilon}\right)_{0<\varepsilon<\varepsilon_{0}}$ be a family of solutions of $(0.7)$ with initial data $\left(\psi_{0}^{\varepsilon}, \eta_{0}^{\varepsilon}\right)$ such that $\left(\nabla \psi^{\varepsilon}, \eta^{\varepsilon}\right)_{0<\varepsilon<\varepsilon_{0}}$ is bounded in $W^{1, \infty}\left(\left[0, \frac{T_{1}}{\varepsilon}\right] ; H^{\sigma}\left(\mathbb{R}^{d}\right)^{d+1}\right)$. We define $V^{\varepsilon}=\nabla \psi^{\varepsilon}$ and choose $(\theta, \lambda, \mu) \in[0,1] \times \mathbb{R}^{2}$ such that the system $\left(T_{\theta, \lambda, \mu}^{1}\right) \in \Sigma$. Then for all $\varepsilon<\varepsilon_{0}$, there exists $T \leq T_{1}$ such that we have:

$$
\forall t \in\left[0, \frac{T}{\varepsilon}\right], \quad\left|V^{\varepsilon}-V_{a p p}^{\varepsilon}\right|_{L^{\infty}\left([0, t] ; H^{s}\right)}+\left|\eta^{\varepsilon}-\eta_{a p p}^{\varepsilon}\right|_{L^{\infty}\left([0, t] ; H^{s}\right)} \leq C \varepsilon^{2} t .
$$

Proof. We follow in this proof the strategy put forward in [8]: estimates are done on the symmetric system that provides the approximate solution rather than on the initial system $(0.7)$.

To this end, we take $(\theta, \lambda, \mu) \in[0,1] \times \mathbb{R}^{2}$ such that the system $T_{\theta, \lambda, \mu}^{1}$ belongs to the class $\Sigma$ of completely symmetric systems.

Since $\left(\psi^{\varepsilon}, \eta^{\varepsilon}\right)_{0<\varepsilon<\varepsilon_{0}}$ is a family of solutions of $(0.7)$ such that $\left(\nabla \psi^{\varepsilon}, \eta^{\varepsilon}\right)_{0<\varepsilon<\varepsilon_{0}}$ is bounded with respect to $\varepsilon$ in $W^{1, \infty}\left(\left[0, \frac{T_{1}}{\varepsilon}\right] ; H^{\sigma}\left(\mathbb{R}^{d}\right)^{d+1}\right)$, using Proposition 2.1 implies that $\left(V_{\theta}^{\varepsilon}, \eta^{\varepsilon}\right)_{0<\varepsilon<\varepsilon_{0}}$ where $V_{\theta}^{\varepsilon}=\left(1+\frac{\varepsilon}{2}\left(1-\theta^{2}\right) \Delta\right) V^{\varepsilon}$ is consistent with the system $S_{\theta, \lambda, \mu}^{1}$.

Moreover, Proposition 2.4 states that any family $\left(V^{\varepsilon}, \eta^{\varepsilon}\right)_{0<\varepsilon<\varepsilon_{0}}$ consistent with the system $S_{\theta, \lambda, \mu}^{1}$ is, up to the aforementionned nonlinear change of variables, consistent with the system $T_{\theta, \lambda, \mu}^{1}$. Applying this result to $\left(V_{\theta}^{\varepsilon}, \eta^{\varepsilon}\right)_{0<\varepsilon<\varepsilon_{0}}$ shows that the family $\left(\tilde{V}_{\theta}^{\varepsilon}, \eta^{\varepsilon}\right)_{0<\varepsilon<\varepsilon_{0}}$, where $\tilde{V}_{\theta}^{\varepsilon}=\left(1+\frac{\varepsilon}{2}\left(\eta^{\varepsilon}-b\right)\right) V_{\theta}^{\varepsilon}$, is actually consistent with the symmetric system $T_{\theta, \lambda, \mu}^{1}$.

Thanks to Proposition 2.6, we know that there exists a time $T_{0}$ such that there exists a unique solution $\left(V_{\Sigma}^{\varepsilon}, \eta_{\Sigma}^{\varepsilon}\right)$ to this system with initial data $\left(V_{\Sigma, 0}^{\varepsilon}, \eta_{\Sigma, 0}^{\varepsilon}\right)$ (defined in the previous formal construction of the approximate solution). We are now interested in computing the error estimates between $\left(\tilde{V}_{\theta}^{\varepsilon}, \eta^{\varepsilon}\right)$ and $\left(V_{\Sigma}^{\varepsilon}, \eta_{\Sigma}^{\varepsilon}\right)$. To this end we define $V=\tilde{V}_{\theta}^{\varepsilon}-V_{\Sigma}^{\varepsilon}$ and $\eta=\eta^{\varepsilon}-\eta_{\Sigma}^{\varepsilon}$. Writing the equations satisfied by $V$ and $\eta$ and performing standard energy estimates on it leads to the following estimate:

$$
\forall t \in\left[0, \frac{T_{1}}{\varepsilon}\right], \quad|V|_{L^{\infty}\left([0, t] ; H^{s}\right)}+|\eta|_{L^{\infty}\left([0, t] ; H^{s}\right)} \leq C \varepsilon^{2} t
$$

where $T=\min \left(T_{0}, T_{1}\right)$. Inverting the nonlinear change of variables and the pseudo-differential one yields the final result. 


\section{Remarks 2.8.}

- The construction of the approximated solution of the water waves problem relies on the choice of the three parameters $\theta, \lambda, \mu$ such that the system $T_{\theta, \lambda, \mu}^{1}$ is completely symmetric. A great advantage of this method is that this choice is totally free: we are indeed allowed to choose any suitable triplet $(\theta, \lambda, \mu)$ we want, and contruct our approximate solution from the exact solution of the system $T_{\theta, \lambda, \mu}^{1}$. In other words, approximate solutions of the water waves problem can be constructed starting from the exact solution of any symmetric system of the class $\Sigma$.

- Our theorem relies implicitly on the existence of a family $\left(\psi^{\varepsilon}, \eta^{\varepsilon}\right)_{0<\varepsilon<\varepsilon_{0}}$ of solutions to the water waves problem in Sobolev spaces: in 1D-surface, this existence have been already proved by Craig [11], Schneider and Wayne [27] thus this implicit hypothesis of existence of solutions is actually a fact. However, in 2D-surface, we have no existence result for the water waves problem on a large time scale. Lannes proved reccenlty in [19] the existence of solutions to this problem in Sobolev spaces in 2D-surface, but we do not know if these solutions persist on a large time scale. Consequently, the analysis is not totally complete in $2 \mathrm{D}$-surface ${ }^{2}$.

\section{THE REGIME OF STRONG TOPOGRAPHY VARIATIONS}

In this section, attention is given to the regime of strong topography variations $\beta=O(1)$. We recall the Boussinesq-like system $\left(\mathcal{B}_{2}\right)$ derived in the previous section, and the fact that solutions of the water waves problem are consistent with this system.

$$
\left(\mathcal{B}_{2}\right)\left\{\begin{array}{l}
\partial_{t} V+\nabla \eta+\frac{\varepsilon}{2} \nabla|V|^{2}=0, \\
\partial_{t} \eta+\nabla \cdot(h V)+\varepsilon\left[\nabla \cdot(\eta V)-\frac{1}{2} \nabla \cdot\left(\frac{h^{3}}{3} \nabla \nabla \cdot V-h^{2} \nabla \nabla \cdot(h V)\right)\right]=0 .
\end{array}\right.
$$

Like in the previous section, we aim at deriving asymptotic models, constructing approximate solutions of the water waves problem, and justifying these approximations. However, the method introduced in the previous section cannot be applyied in the exact same way: this regime is indeed much more complex since the bottom terms have here a greater influence than in the first regime. These bottom terms introduce new difficulties which compell us to revise and adapt our strategy.

\subsection{A first equivalent system}

First remark that the bottom term $h$ (recall that $h=1-b$ is the non-dimensional still water depth) appears in the first order term of the second equation of $\left(\mathcal{B}_{2}\right)$ whereas it is not present in the first one. This fact becomes important when it comes to the BBM trick which is unlikely to symetrize these terms. To correctly deal with this regime, we have to invert the order of the change of variables, and proceed with an adapted nonlinear change of variables first that symetrizes both order one terms and non-linear terms.

Taking into account the fact that we have to symmetrize both order one terms and nonlinear terms, we introduce the following change of variables:

$$
\tilde{V}=\left(\sqrt{h}+\frac{\varepsilon}{2} \frac{\eta}{\sqrt{h}}\right) V
$$

so that

$$
V=\left(\frac{1}{\sqrt{h}}-\frac{\varepsilon}{2} \frac{\eta}{h \sqrt{h}}\right) \tilde{V}+O\left(\varepsilon^{2}\right)
$$

\footnotetext{
${ }^{2}$ In [2], Alvarez-Samaniego and Lannes proved the well posedness result assumed here in any dimension, and thus completed the justification of the present Boussinesq-like systems with uneven bottoms.
} 
Assuming that $\nabla \times \tilde{V}=O(\varepsilon)$, we formally derive the following system of equations satisfied by $\tilde{V}$ and $\eta$ :

$$
\left\{\begin{array}{c}
\partial_{t} \tilde{V}+\sqrt{h} \nabla \eta+\frac{\varepsilon}{2 \sqrt{h}}\left[\frac{1}{2} \nabla \eta^{2}+\frac{1}{2} \nabla|\tilde{V}|^{2}+(\tilde{V} \cdot \nabla) \tilde{V}+\tilde{V} \nabla \cdot \tilde{V}\right. \\
\left.+\frac{1}{h}\left(\frac{1}{2}(\nabla h \cdot \tilde{V}) \tilde{V}-|\tilde{V}|^{2} \nabla h\right)\right]=O\left(\varepsilon^{2}\right), \\
\partial_{t} \eta+\nabla(\sqrt{h} \cdot \tilde{V})+\frac{\varepsilon}{2 \sqrt{h}}\left[\nabla \cdot(\eta \tilde{V})-\sqrt{h} \nabla \cdot\left(\frac{h^{3}}{3} \nabla \nabla \cdot\left(\frac{\tilde{V}}{\sqrt{h}}\right)\right.\right. \\
\left.\left.-h^{2} \nabla \nabla \cdot(\sqrt{h} \tilde{V})\right)-\frac{\eta}{2 h} \nabla h \cdot \tilde{V}\right]=O\left(\varepsilon^{2}\right) .
\end{array}\right.
$$

We introduce the system $\left(\mathcal{T}_{b}\right)$ that corresponds to the homogeneous version of the previous system:

$$
\left(\mathcal{T}_{b}\right)\left\{\begin{array}{l}
\partial_{t} V+\sqrt{h} \nabla \eta+\frac{\varepsilon}{2} F_{h}\left(\begin{array}{c}
V \\
\eta
\end{array}\right)=0, \\
\partial_{t} \eta+\nabla(\sqrt{h} \cdot V)+\frac{\varepsilon}{2}\left[f_{h}\left(\begin{array}{c}
V \\
\eta
\end{array}\right)-\nabla \cdot\left(\frac{h^{3}}{3} \nabla \nabla \cdot\left(\frac{V}{\sqrt{h}}\right)-h^{2} \nabla \nabla \cdot(\sqrt{h} V)\right)\right]=0
\end{array}\right.
$$

where

$$
\left\{\begin{aligned}
F_{h}\left(\begin{array}{c}
V \\
\eta
\end{array}\right) & =\frac{1}{\sqrt{h}}\left(\frac{1}{2} \nabla \eta^{2}+\frac{1}{2} \nabla|V|^{2}+(V \cdot \nabla) V+V \nabla \cdot V+\frac{1}{h}\left(\frac{1}{2}(\nabla h \cdot V) V-|V|^{2} \nabla h\right)\right) \\
f_{h}\left(\begin{array}{c}
V \\
\eta
\end{array}\right) & =\frac{1}{\sqrt{h}}\left(\nabla \cdot(\eta V)-\frac{\eta}{2 h} \nabla h \cdot V\right)
\end{aligned}\right.
$$

On this new system $\left(\mathcal{T}_{b}\right)$, we have the following consistency result:

Proposition 3.1. Consider a family $\left(\psi^{\varepsilon}, \eta^{\varepsilon}\right)_{0<\varepsilon<\varepsilon_{0}}$ of solutions of $(0.7)$ such that $\left(\nabla \psi^{\varepsilon}, \eta^{\varepsilon}\right)_{0<\varepsilon<\varepsilon_{0}}$ is bounded with respect to $\varepsilon$ in $W^{1, \infty}\left(\left[0, \frac{T}{\varepsilon}\right] ; H^{\sigma}\left(\mathbb{R}^{d}\right)^{d+1}\right)$ with $\sigma$ large enough. Then the family $\left(V^{\varepsilon}, \eta^{\varepsilon}\right)_{0<\varepsilon<\varepsilon_{0}}$ is consistent with the system $\left(\mathcal{T}_{b}\right)$, where $V^{\varepsilon}=\nabla \psi^{\varepsilon}$.

Proof. First remark that since the velocity field $V^{\varepsilon}$ is irrotationnal, we have $\nabla \times \tilde{V}^{\varepsilon}=O(\varepsilon)$. And since $\left(\nabla \psi^{\varepsilon}, \eta^{\varepsilon}\right)_{0<\varepsilon<\varepsilon_{0}}$ is consistent with the Boussinesq-like system $\left(\mathcal{B}_{2}\right)$, the previous computations yield directly the result.

\subsection{Derivation of a class of equivalent systems}

In the previous section, we saw that a suitable change of variable comes from considering $V_{\theta}$, the horizontal component of the velocity at the height $-1+\theta(\theta \in[0,1])$, instead of the horizontal component of the velocity field at the free surface. We can remark that the link between these two variables (and hence the adequate change of variables) can be derived from the expression of $u_{a p p}$ computed during the asymptotic expansion process of the operator $Z_{\varepsilon}(\varepsilon \eta, \beta b)$, which implies that we must adapt the change of variable for the strong variations regime since the expression of $u_{a p p}$ now strongly depends on the topography.

We saw in the previous section that the computation of the asymptotic expansion of $Z_{\varepsilon}(\eta, b) \psi$ relies on finding an approximate solution of the elliptic problem $(H)$ on the band $\mathcal{S}=[-1,0] \times \mathbb{R}^{2}$. Starting from the truncation of the computed value of $u_{a p p}$ at the order $O\left(\varepsilon^{2}\right)$,

$$
u_{a p p}=\psi+\varepsilon\left[\left(\frac{1}{2}-\frac{(z+1)^{2}}{2}\right) h^{2} \Delta \psi-z h \nabla h \cdot \nabla \psi\right]+O\left(\varepsilon^{2}\right)
$$


where $\psi$ is the value of the velocity potential at the free surface, shows that $\nabla u_{a p p}(\cdot, z)$ gives an approximation at order $\varepsilon^{2}$ of the horizontal component of the velocity field, namely $V(\cdot, z)=\nabla \phi(\cdot, z)$ at height $z \in[-1,0]$.

Consequently, in presence of large bottom variations, the adequate change of variables is given by:

$$
V_{\theta}=\left[1-\frac{\varepsilon}{2}(\theta-1)\left(\theta \nabla\left(h^{2} \nabla \cdot\right)+\nabla \nabla \cdot\left(h^{2} \cdot\right)\right)\right] V,
$$

so that

$$
V=\left[1+\frac{\varepsilon}{2}(\theta-1)\left(\theta \nabla\left(h^{2} \nabla \cdot\right)+\nabla \nabla \cdot\left(h^{2} \cdot\right)\right)\right] V_{\theta}+O\left(\varepsilon^{2}\right) .
$$

From this change of variables, we easily compute the expressions of $\partial_{t} V$ and $\nabla \cdot \sqrt{h} V$ which we plug into the system $\left(\mathcal{T}_{b}\right)$. By rewriting carefully the topography terms in order to make the quantity $\sqrt{h}$ appear, one gets the following system:

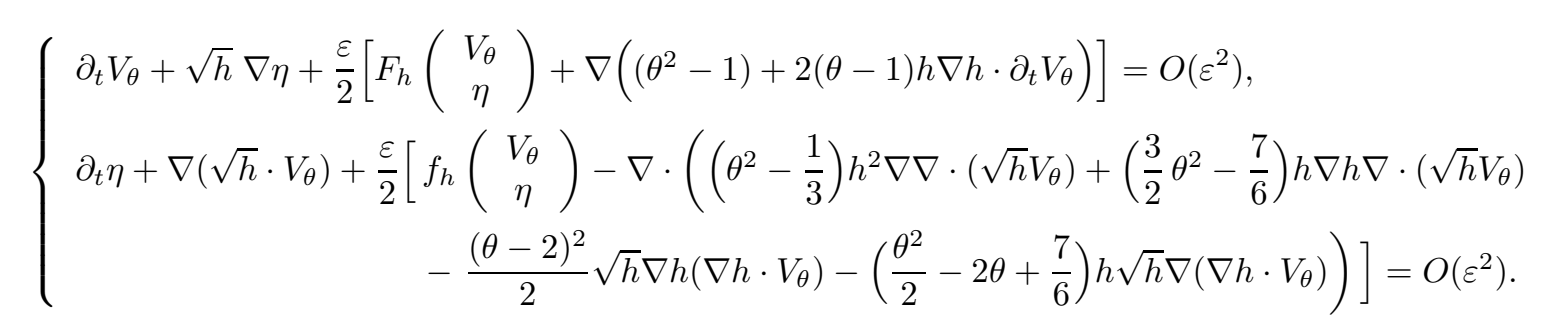

At this point a new problem arises. Applying the BBM trick in the exact same way as in the previous regime leads to a system that is never symmetric for any values of the parameters $\theta, \lambda$ and $\mu$. Indeed, this requires the resolution of an over-determined numerical system on the unknowns $\theta, \lambda$ and $\mu$. To handle this problem, we simply introduce an additionnal unknown during the BBM trick process:

$$
\left\{\begin{array}{l}
\partial_{t} V_{\theta}=\left(1-\lambda_{1}\right) \partial_{t} V_{\theta}-\lambda_{1} \sqrt{h} \nabla \eta+O(\varepsilon), \\
\partial_{t} V_{\theta}=\left(1-\lambda_{2}\right) \partial_{t} V_{\theta}-\lambda_{2} \sqrt{h} \nabla \eta+O(\varepsilon) \\
\nabla \cdot\left(\sqrt{h} V_{\theta}\right)=\mu \nabla \cdot\left(\sqrt{h} V_{\theta}\right)-(1-\mu) \partial_{t} \eta+O(\varepsilon),
\end{array}\right.
$$

where we use the first relation on the term $\left(\theta^{2}-1\right) h^{2} \nabla \cdot \partial_{t} V_{\theta}$ and the second relation on the term $2(\theta-1) h \nabla h \cdot \partial_{t} V_{\theta}$.

Finally, we formally derive a new class $\left(\mathcal{S}_{b}\right)$ of systems, and we can prove that if a family $\left(V^{\varepsilon}, \eta^{\varepsilon}\right)_{0<\varepsilon<\varepsilon_{0}}$ is consistent with the system $\Gamma_{b}$ then $\left(V_{\theta}^{\varepsilon}, \eta^{\varepsilon}\right)_{0<\varepsilon<\varepsilon_{0}}$ - where $V_{\theta}^{\varepsilon}=\left[1-\frac{\varepsilon}{2}(\theta-1)\left(\theta \nabla\left(h^{2} \nabla \cdot\right)+\nabla \nabla \cdot\left(h^{2}.\right)\right)\right] V^{\varepsilon}-$ is consistent with any of the following systems $\left(S_{\theta, \lambda_{1}, \lambda_{2}, \mu}\right)$ :

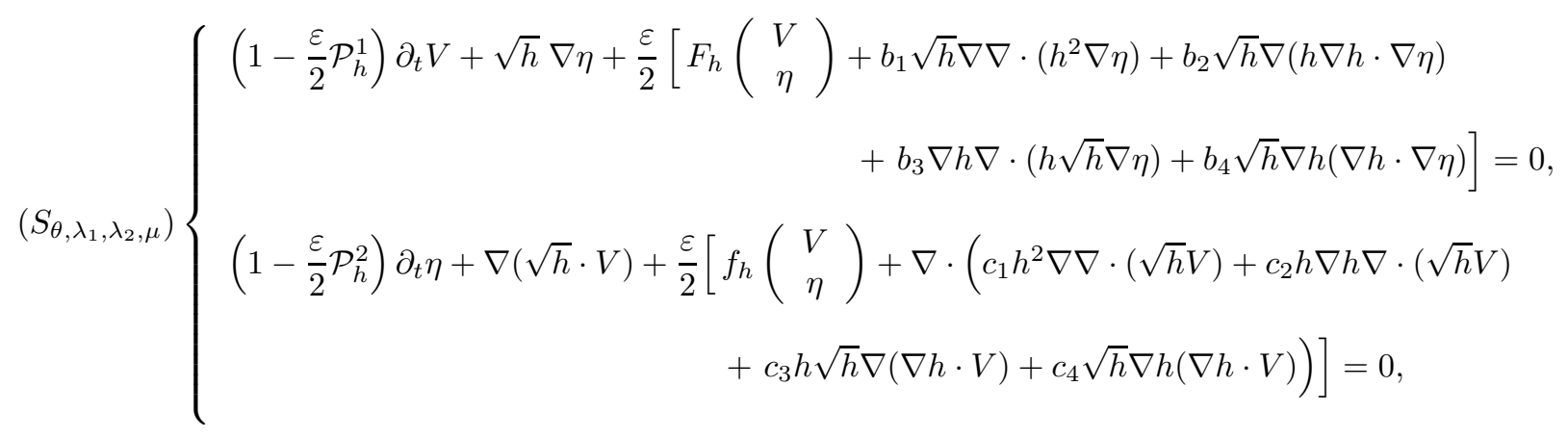


where the operators $\mathcal{P}_{h}^{1}$ and $\mathcal{P}_{h}^{2}$ are defined by

$$
\left\{\begin{array}{l}
\mathcal{P}_{h}^{1}=(1-\theta)\left(\left(1-\lambda_{1}\right)(\theta+1) \nabla\left(h^{2} \nabla \cdot \quad\right)+2\left(1-\lambda_{2}\right) \nabla(h \nabla h \cdot \quad)\right), \\
\mathcal{P}_{h}^{2}=(1-\mu)\left(\left(\theta^{2}-\frac{1}{3}\right) \nabla \cdot\left(h^{2} \nabla \quad\right)+\left(\frac{3}{2} \theta^{2}-\frac{7}{6}\right) \nabla \cdot(h \nabla h \times \quad)\right),
\end{array}\right.
$$

and the parameters $\left(a_{i}\right)_{1 \leq i \leq 4},\left(b_{i}\right)_{1 \leq i \leq 4}$ have the following expressions:

$$
\left\{\begin{array}{lll}
b_{1}=\lambda_{1}\left(1-\theta^{2}\right) ; & c_{1}=\mu\left(\theta^{2}-\frac{1}{3}\right) \\
b_{2}=(1-\theta)\left(2 \lambda_{2}-\frac{3}{2} \lambda_{1}(1+\theta)\right) ; & c_{2}=\mu\left(\frac{3}{2} \theta^{2}-\frac{7}{6}\right) \\
b_{3}=\frac{\lambda_{1}}{2}\left(1-\theta^{2}\right) ; & c_{3}=-\frac{1}{2} \theta^{2}+2 \theta-\frac{7}{6} \\
b_{4}=(1-\theta)\left(\lambda_{2}-\frac{\lambda_{1}}{2}(1+\theta)\right) ; & c_{4}=\frac{1}{2}(\theta-2)^{2} .
\end{array}\right.
$$

The previous computations are summed up in the following proposition.

Proposition 3.2. Let $\theta \in[0,1]$ and $\left(\psi^{\varepsilon}, \eta^{\varepsilon}\right)_{0<\varepsilon<\varepsilon_{0}}$ a family of solutions of $(0.7)$ such that $\left(\nabla \psi^{\varepsilon}, \eta^{\varepsilon}\right)_{0<\varepsilon<\varepsilon_{0}}$ is bounded with respect to $\varepsilon$ in $W^{1, \infty}\left(\left[0, \frac{T}{\varepsilon}\right] ; H^{\sigma}\left(\mathbb{R}^{d}\right)^{d+1}\right)$ with $\sigma$ large enough. We define $V^{\varepsilon}=\nabla \psi^{\varepsilon}$ and $\tilde{V}^{\varepsilon}=\left(1-\frac{\varepsilon}{2}(\theta-1)\left(\theta \nabla\left(h^{2} \nabla \cdot\right)+\nabla \nabla \cdot\left(h^{2} \cdot\right)\right)\right)\left(\sqrt{h}+\frac{\varepsilon}{2} \frac{\eta}{\sqrt{h}}\right) V^{\varepsilon}$. Then for all $\left(\lambda_{1}, \lambda_{2}, \mu\right) \in \mathbb{R}^{3}$, the family $\left(\tilde{V}^{\varepsilon}, \eta^{\varepsilon}\right)_{0<\varepsilon<\varepsilon_{0}}$ is consistent with the system $\left(S_{\theta, \lambda_{1}, \lambda_{2}, \mu}\right)$.

Moreover, we have the following proposition on the existence of a subclass of $\left(\mathcal{S}_{b}\right)$ composed with fully symmetric systems.

Proposition 3.3. There exists at least one value of $\left(\theta, \lambda_{1}, \lambda_{2}, \mu\right)$ such that the system $\left(S_{\theta, \lambda_{1}, \lambda_{2}, \mu}\right)$ is fully symmetric.

Proof. We are concerned here with the resolution of the following system:

$$
\left\{\begin{array}{l}
b_{1}=c_{1} \\
b_{2}=-c_{2} \\
b_{3}=c_{3} \\
b_{4}=-c_{4}
\end{array}\right.
$$

This system on $\left(\theta, \lambda_{1}, \lambda_{2}, \mu\right)$ have at least one solution that gives the following approximate values:

$$
\left\{\begin{array}{l}
\theta \approx 0.6318 \\
\lambda_{1} \approx-0.3416 \\
\lambda_{2} \approx-2.8209 \\
\mu \approx-3.1157
\end{array}\right.
$$

which ends the proof.

From now on, we only consider this solution and its approximate values.

\subsection{The fully symmetric systems}

Thanks to Proposition 3.3, we know that some of the systems $\left(S_{\theta, \lambda_{1}, \lambda_{2}, \mu}\right)$ of the class $\left(S_{b}\right)$ are completely symmetric: we hence denote by $\Sigma_{b}$ the non-empty subclass of $\left(S_{b}\right)$ composed with these symmetric systems. Unfortunately, we do not have the same kind of existence theory on these systems as in the previous regime. 
Indeed, the main difference consists in the order one terms of the two equations $\left(\begin{array}{c}\sqrt{h} \nabla \eta \\ \nabla \cdot(\sqrt{h} V)\end{array}\right)$. In order to focus on the problem, we rewrite these terms: $A\left(X, \partial_{X}\right)\left(\begin{array}{c}V \\ \eta\end{array}\right)$ where $A\left(X, \partial_{X}\right)=\left(\begin{array}{cc}0 & \sqrt{h} \nabla_{X} \\ \nabla_{X} \cdot(\sqrt{h} \times) & 0\end{array}\right)$. The proof of the existence of solutions on a short time scale is not modified by these terms, the classical proof is still valid. However, the fact that the matrix $A$ depends on the bottom term $h$ is a real problem as far as the large time existence is concerned: indeed, one crucial point of the proof here relies on the size of the quantity $\frac{\nabla h}{\varepsilon}$ on which we have no piece of information. The only case wherein we are surely able to demonstrate the large time existence is the case where $\nabla h$ is of order $O(\varepsilon)$ : the term $\frac{\nabla h}{\varepsilon}$ is then of order $O(1)$ and we can conclude. In all other cases, the classical proof fails to provide a rigourous demonstration of the long time existence of solutions to these symmetric systems. Nevertheless, we are able to state the following proposition:

Proposition 3.4. Let $s>\frac{d}{2}+1$ and $\left(\theta, \lambda_{1}, \lambda_{2}, \mu\right)$ be such that the system $\left(S_{\theta, \lambda_{1}, \lambda_{2}, \mu}\right)$ belongs to the class $\Sigma_{b}$.

Then for all $\left(V_{0}, \eta_{0}\right) \in H^{s}\left(\mathbb{R}^{d}\right)^{d+1}$, there exists a time $T_{0}$ independant of $\varepsilon$ and a unique solution $(V, \eta) \in$ $C\left(\left[0, T_{0}\right] ; H^{s}\left(\mathbb{R}^{d}\right)^{d+1}\right) \cap C^{1}\left(\left[0, T_{0}\right] ; H^{s-3}\left(\mathbb{R}^{d}\right)^{d+1}\right)$ to the system $\left(S_{\theta, \lambda_{1}, \lambda_{2}, \mu}\right)$ such that $(V, \eta)_{\left.\right|_{t=0}}=\left(V_{0}, \eta_{0}\right)$.

Furthermore, this unique solution is bounded independently of $\varepsilon$ in the following sense: there exists a constant $C_{0}$ independent of $\varepsilon$ such that for all $k$ verifying $s-3 k>\frac{d}{2}+1$, we have:

$$
|(V, \eta)|_{W^{k, \infty}\left(\left[0, T_{0}\right] ; H^{s-3 k}\left(\mathbb{R}^{d}\right)^{d+1}\right)} \leq C_{0} .
$$

Besides, if we suppose that $\nabla h=O(\varepsilon)$, the previous result becomes valid on the long time interval $\left[0, \frac{T_{0}}{\varepsilon}\right]$.

Proof. The key point of the proof is to demonstrate that the elliptic operator $1-\frac{\varepsilon}{2}\left(\begin{array}{c}\mathcal{P}_{h}^{1} \\ \mathcal{P}_{h}^{2}\end{array}\right)$ is a positive one. We first focus on $\mathcal{P}_{h}^{1}$ :

$$
\left(1-\frac{\varepsilon}{2} \mathcal{P}_{h}^{1} V, V\right)=|V|_{2}^{2}+\frac{\varepsilon}{2}\left(1-\theta^{2}\right)\left(1-\lambda_{1}\right)|h \nabla \cdot V|_{2}^{2}+\varepsilon(1-\theta)\left(1-\lambda_{2}\right)(\nabla h \cdot V, h \nabla \cdot V) .
$$

Using the following inequality (satisfied for all $a \in \mathbb{R}$ ):

$$
|(\nabla h \cdot V, h \nabla \cdot V)| \leq \frac{a^{2}}{2}|h \nabla \cdot V|_{2}^{2}+\frac{1}{2 a^{2}}|\nabla h \cdot V|_{2}^{2},
$$

and taking $a^{2}=\frac{(1+\theta)\left(1-\lambda_{1}\right)}{1-\lambda_{2}}$ leads to:

$$
\left(1-\frac{\varepsilon}{2} \mathcal{P}_{h}^{1} V, V\right) \geq|V|_{2}^{2}-\frac{\varepsilon}{2} \frac{(1-\theta)\left(1-\lambda_{2}\right)^{2}}{(1+\theta)\left(1-\lambda_{1}\right)}|\nabla h \cdot V|_{2}^{2} .
$$

Using the classical Cauchy-Schwarz inequality leads finally to:

$$
\left(1-\frac{\varepsilon}{2} \mathcal{P}_{h}^{1} V, V\right) \geq\left(1-\frac{\varepsilon}{2} \frac{(1-\theta)\left(1-\lambda_{2}\right)^{2}}{(1+\theta)\left(1-\lambda_{1}\right)}|\nabla h|_{2}^{2}\right)|V|_{2}^{2} .
$$

At this point, if we take a small enough value of $\varepsilon$, f.e. $\varepsilon \leq \frac{2(1+\theta)\left(1-\lambda_{1}\right)}{(1-\theta)\left(1-\lambda_{2}\right)^{2}|\nabla h|_{2}^{2}}$, it ensures the global positivity of $\mathcal{P}_{h}^{1}$. On $\mathcal{P}_{h}^{2}$, we use the same method:

$$
\left(1-\frac{\varepsilon}{2} \mathcal{P}_{h}^{2} \eta, \eta\right)=|\eta|_{2}^{2}+\frac{\varepsilon}{2}(1-\mu)\left(\theta^{2}-\frac{1}{3}\right)|h \nabla \eta|_{2}^{2}+\frac{\varepsilon}{2}(1-\mu)\left(\frac{3}{2} \theta^{2}-\frac{7}{6}\right)(\eta \nabla h, h \nabla \eta) .
$$

Using the same ideas as previously, one gets:

$$
\left(1-\frac{\varepsilon}{2} \mathcal{P}_{h}^{2} \eta, \eta\right) \geq\left(1-\frac{\varepsilon}{8} \frac{(1-\mu)\left(\frac{3}{2} \theta^{2}-\frac{7}{6}\right)^{2}}{\theta^{2}-\frac{1}{3}}|\nabla h|_{2}^{2}\right)|\eta|_{2}^{2} .
$$


Once more, if we take f.e. $\varepsilon \leq \frac{8\left(\theta^{2}-\frac{1}{3}\right)}{(1-\mu)\left(\frac{3}{2} \theta^{2}-\frac{7}{6}\right)^{2}|\nabla h|_{2}^{2}}$, we have the global positivity of $\mathcal{P}_{h}^{2}$.

Consequently, taking $\varepsilon \leq \min \left(\frac{2(1+\theta)\left(1-\lambda_{1}\right)}{(1-\theta)\left(1-\lambda_{2}\right)^{2}|\nabla h|_{2}^{2}}, \frac{8\left(\theta^{2}-\frac{1}{3}\right)}{(1-\mu)\left(\frac{3}{2} \theta^{2}-\frac{7}{6}\right)^{2}|\nabla h|_{2}^{2}}\right)$ ensures that the operator $1-\frac{\varepsilon}{2}\left(\begin{array}{c}\mathcal{P}_{h}^{1} \\ \mathcal{P}_{h}^{2}\end{array}\right)$ is positive.

At this point, using this result and performing usual energy estimates on the system proves the existence of a time $T$ such that there exists an unique solution $(V, \eta) \in C\left([0, T] ; H^{s}\left(\mathbb{R}^{d}\right)^{d+1}\right) \cap C^{1}\left([0, T] ; H^{s-3}\left(\mathbb{R}^{d}\right)^{d+1}\right)$ to the system.

This result gives an efficient theoretical background to contruct approximate solutions of the water waves problem on a time scale $O(1)$, and $O\left(\frac{1}{\varepsilon}\right)$ in the case $\nabla h=O(\varepsilon)$.

This contruction follows the same steps — but in a different order — as the contruction of approximate solutions for the first regime: we consider a solution $\left(\psi^{\varepsilon}, \eta^{\varepsilon}\right)$ to the formulation $(0.7)$ of the water waves problem. We take initial data $\left(\psi_{0}^{\varepsilon}, \eta_{0}^{\varepsilon}\right)$ such that $\left(\nabla \psi_{0}^{\varepsilon}, \eta_{0}^{\varepsilon}\right) \in H^{s}\left(\mathbb{R}^{d}\right)^{d+1}$ for a suitably large value of $s$. We then define $V^{\varepsilon}=\nabla \psi^{\varepsilon}$ and $V_{0}^{\varepsilon}=\nabla \psi_{0}^{\varepsilon}$ : we first contruct the data $\left(V_{\Sigma, 0}^{\varepsilon}, \eta_{\Sigma, 0}^{\varepsilon}\right)$ by applying the two successive changes of variable on the data $\left(V_{0}^{\varepsilon}, \eta_{0}^{\varepsilon}\right)$. We then choose the parameters $\left(\theta, \lambda_{1}, \lambda_{2}, \mu\right) \in[0,1] \times \mathbb{R}^{3}$ such that the system $\left(S_{\theta, \lambda_{1}, \lambda_{2}, \mu}\right)$ is completely symmetric. Using Proposition 3.4, we know that there exists a unique solution to this system with initial data $\left(V_{\Sigma, 0}^{\varepsilon}, \eta_{\Sigma, 0}^{\varepsilon}\right)$ : we denote this solution by $\left(V_{\Sigma}^{\varepsilon}, \eta_{\Sigma}^{\varepsilon}\right)$. From this exact solution of the symmetric system $\left(S_{\theta, \lambda_{1}, \lambda_{2}, \mu}\right)$, we finally construct an approximate solution of the water waves problem by successively and approximatively inverting the two changes of variable as shown below (which is possible if $\varepsilon$ is small enough):

$$
\left\{\begin{array}{l}
V_{a p p}^{\varepsilon}=\left(\frac{1}{\sqrt{h}}-\frac{\varepsilon}{2} \frac{\eta^{\varepsilon}}{h \sqrt{h}}\right)\left(1+\frac{\varepsilon}{2}(\theta-1)\left(\theta \nabla\left(h^{2} \nabla \cdot V_{\Sigma}^{\varepsilon}\right)+\nabla \nabla \cdot\left(h^{2} V_{\Sigma}^{\varepsilon}\right)\right)\right) \\
\eta_{a p p}^{\varepsilon}=\eta_{\Sigma}^{\varepsilon} .
\end{array}\right.
$$

We are now able to state our final result:

Theorem 3.5. Let $T_{1} \geq 0, s \geq \frac{d}{2}+1, \sigma \geq s+3$ and $\left(\nabla \psi_{0}^{\varepsilon}, \eta_{0}^{\varepsilon}\right)$ be in $H^{\sigma}\left(\mathbb{R}^{d}\right)^{d+1}$. Let $\left(\psi^{\varepsilon}, \eta^{\varepsilon}\right)_{0<\varepsilon<\varepsilon_{0}}$ be a family of solutions of (0.7) with initial data $\left(\psi_{0}^{\varepsilon}, \eta_{0}^{\varepsilon}\right)$ such that $\left(\nabla \psi^{\varepsilon}, \eta^{\varepsilon}\right)_{0<\varepsilon<\varepsilon_{0}}$ is bounded in $W^{1, \infty}\left(\left[0, T_{1}\right] ; H^{\sigma}\left(\mathbb{R}^{d}\right)^{d+1}\right)$. We define $V^{\varepsilon}=\nabla \psi^{\varepsilon}$ and choose $\left(\theta, \lambda_{1}, \lambda_{2}, \mu\right) \in[0,1] \times \mathbb{R}^{3}$ such that the system $\left(S_{\theta, \lambda_{1}, \lambda_{2}, \mu}\right) \in \Sigma_{b}$.

Then for all $\varepsilon<\varepsilon_{0}$, there exists a time $T \leq T_{1}$ such that we have:

$$
\left|V^{\varepsilon}-V_{a p p}^{\varepsilon}\right|_{L^{\infty}\left([0, T] ; H^{s}\right)}+\left|\eta^{\varepsilon}-\eta_{a p p}^{\varepsilon}\right|_{L^{\infty}\left([0, T] ; H^{s}\right)} \leq C \varepsilon^{2}
$$

Besides, if we suppose that $\nabla h=O(\varepsilon)$ then $\left(V_{a p p}^{\varepsilon}, \eta_{a p p}^{\varepsilon}\right)$ approximates the water waves solutions on a large time scale:

$$
\forall t \in\left[0, \frac{T}{\varepsilon}\right], \quad\left|V^{\varepsilon}-V_{a p p}^{\varepsilon}\right|_{L^{\infty}\left([0, t] ; H^{s}\right)}+\left|\eta^{\varepsilon}-\eta_{a p p}^{\varepsilon}\right|_{L^{\infty}\left([0, t] ; H^{s}\right)} \leq C \varepsilon^{2} t .
$$

Proof. The proof is an adaptation of the one of Theorem 2.7, and we omit it here.

Remark 3.6. In the general case, where we have no piece of information on the size of the quantity $\frac{\nabla h}{\varepsilon}$, our analysis is complete on a short time scale. We have indeed an approximation on this interval of time, and we know from Lannes [19] the existence of solutions to the water waves problem on a short time scale in $2 \mathrm{D}$ and $3 \mathrm{D}$. However, in the case $\nabla h=O(\varepsilon)$, this analysis is only complete in $2 \mathrm{D}$ - like in the first regime - since we do not know about the existence of solutions to the water waves problem on a large time scale.

Remark 3.7. The regime of long wave $\left(\varepsilon=\mu \ll 1\right.$ where $\left.\mu=\frac{h^{2}}{\lambda^{2}}\right)$ and strong topography variations $(\beta=O(1))$ considered here can be seen as a particular case of the Green-Naghdi regime $(\mu \ll 1$ and no particular assumption on $\varepsilon$ and $\beta$ ) derived in [14] and fully justified in $[2,3]$. 
Acknowledgements. This work was supported by the ACI Jeunes chercheurs du ministère de la Recherche "Dispersion et nonlinéarités".

\section{REFERENCES}

[1] S. Alinhac and P. Gérard, Opérateurs pseudo-différentiels et théorème de Nash-Moser. Savoirs Actuels, InterEditions, Paris, Editions du Centre National de la Recherche Scientifique (CNRS), Meudon (1991) p. 190.

[2] B. Alvarez-Samaniego and D. Lannes, Large time existence for $3 D$ water-waves and asymptotics. Technical report (http://fr.arxiv.org/abs/math/0702015v1), Université Bordeaux I, IMB (2007).

[3] B. Alvarez-Samaniego and D. Lannes, A Nash-Moser theorem for singular evolution equations. Application to the Serre and Green-Naghdi equations. Preprint (http://arxiv.org/abs/math.AP/0701681v1), Indiana University Mathematical Journal (2007) (to appear).

[4] T.B. Benjamin, J.L. Bona and J.J. Mahony, Model equations for long waves in nonlinear dispersive systems. Philos. Trans. Roy. Soc. London Ser. A 272 (1972) 47-78.

[5] J.L. Bona and M. Chen, A Boussinesq system for two-way propagation of nonlinear dispersive waves. Physica D 116 (2004) $191-224$.

[6] J.L. Bona, M. Chen and J.-C. Saut, Boussinesq equations and other systems for small-amplitude long waves in nonlinear dispersive media. I: Derivation and linear theory. J. Nonlinear Sci. 12 (2002) 283-318.

[7] J.L. Bona, M. Chen and J.-C. Saut, Boussinesq equations and other systems for small-amplitude long waves in nonlinear dispersive media. II: Nonlinear theory. Nonlinearity 17 (2004) 925-952.

[8] J.L. Bona, T. Colin and D. Lannes, Long waves approximations for water waves. Arch. Rational Mech. Anal. 178 (2005) 373-410.

[9] M.J. Boussinesq, Théorie de l'intumescence liquide appelée onde solitaire ou de translation se propageant dans un canal rectangulaire. C.R. Acad. Sci. Paris Sér. A-B $\mathbf{7 2}$ (1871) 755-759.

[10] M. Chen, Equations for bi-directional waves over an uneven bottom. Math. Comput. Simulation 62 (2003) 3-9.

[11] W. Craig, An existence theory for water waves and the Boussinesq and Korteweg-de Vries scaling limits. Comm. Partial Differential Equations 10 (1985) 787-1003.

[12] M.W. Dingemans, Water Wave Propagation over uneven bottoms. Part I: Linear Wave Propagation. Adanced Series on Ocean Engineering 13. World Scientific (1997).

[13] M.W. Dingemans, Water Wave Propagation over uneven bottoms. Part II: Non-linear Wave Propagation. Adanced Series on Ocean Engineering 13. World Scientific (1997).

[14] A.E. Green and P.M. Naghdi, A derivation of equations for wave propagation in water of variable depth. J. Fluid Mech. 78 (1976) $237-246$.

[15] T. Iguchi, A long wave approximation for capillary-gravity waves and an effect of the bottom. Preprint (2005).

[16] T. Iguchi, A mathematical justification of the forced Korteweg-de Vries equation for capillary-gravity waves. Kyushu J. Math. 60 (2006) 267-303.

[17] J.T. Kirby, Gravity Waves in Water of Finite Depth, Advances in Fluid Mechanics 10, in J.N. Hunt Ed., Computational Mechanics Publications (1997) 55-125.

[18] D. Lannes, Sur le caractère bien posé des équations d'Euler avec surface libre. Séminaire EDP de l'École Polytechnique (2004), Exposé no. XIV.

[19] D. Lannes, Well-posedness of the water-waves equations. J. Amer. Math. Soc. 18 (2005) 605-654.

$[20]$ D. Lannes and J.C. Saut, Weakly transverse Boussinesq systems and the Kadomtsev-Petviashvili approximation. Nonlinearity 19 (2006) 2853-2875.

[21] P.A. Madsen, R. Murray and O.R. Sorensen, A new form of the Boussinesq equations with improved linear dispersion characteristics (Part 1). Coastal Eng. 15 (1991) 371-388.

[22] G. Métivier, Small Viscosity and Boundary Layer Methods: Theory, Stability Analysis, and Applications. Modeling and Simulation in Science, Engineering and Technology, Birkhäuser, Boston-Basel-Berlin (2004).

[23] D.P. Nicholls and F. Reitich, A new approach to analyticity of Dirichlet-Neumann operators. Proc. Royal Soc. Edinburgh Sect. A 131 (2001) 1411-1433.

[24] V.I. Nalimov, The Cauchy-Poisson problem. (Russian) Dinamika Splošn. Sredy Vyp. 18, Dinamika Zidkost. so Svobod. Granicami 254 (1974) 104-210.

[25] O. Nwogu, Alternative form of Boussinesq equations for nearshore wave propagation. J. Waterw. Port Coastal Eng. ASCE 119 (1993) 618-638.

[26] D.H. Peregrine, Long waves on a beach. J. Fluid Mech. 27 (1967) 815-827.

[27] G. Schneider and C.E. Wayne, The long-wave limit for the water-wave problem. I. The case of zero surface tension. Comm. Pure Appl. Math. 162 (2002) 247-285.

[28] G. Wei and J.T. Kirby, A time-dependent numerical code for extended Boussinesq equations. J. Waterw. Port Coastal Ocean Engineering 120 (1995) 251-261. 
[29] G. Wei, J.T. Kirby, S.T. Grilli and R. Subramanya, A fully nonlinear Boussinesq model for surface waves. I. Highly nonlinear, unsteady waves. J. Fluid Mechanics 294 (1995) 71-92.

[30] S. Wu, Well-posedness in Sobolev spaces of the full water wave problem in 2-D. Invent. Math. 130 (1997) 39-72.

[31] S. Wu, Well-posedness in Sobolev spaces of the full water wave problem in 3-D. J. Amer. Math. Soc. 12 (1999) $445-495$.

[32] H. Yosihara, Gravity waves on the free surface of an incompressible perfect fluid of finite depth. Publ. Res. Inst. Math. Sci. 18 (1982) 49-96. 